
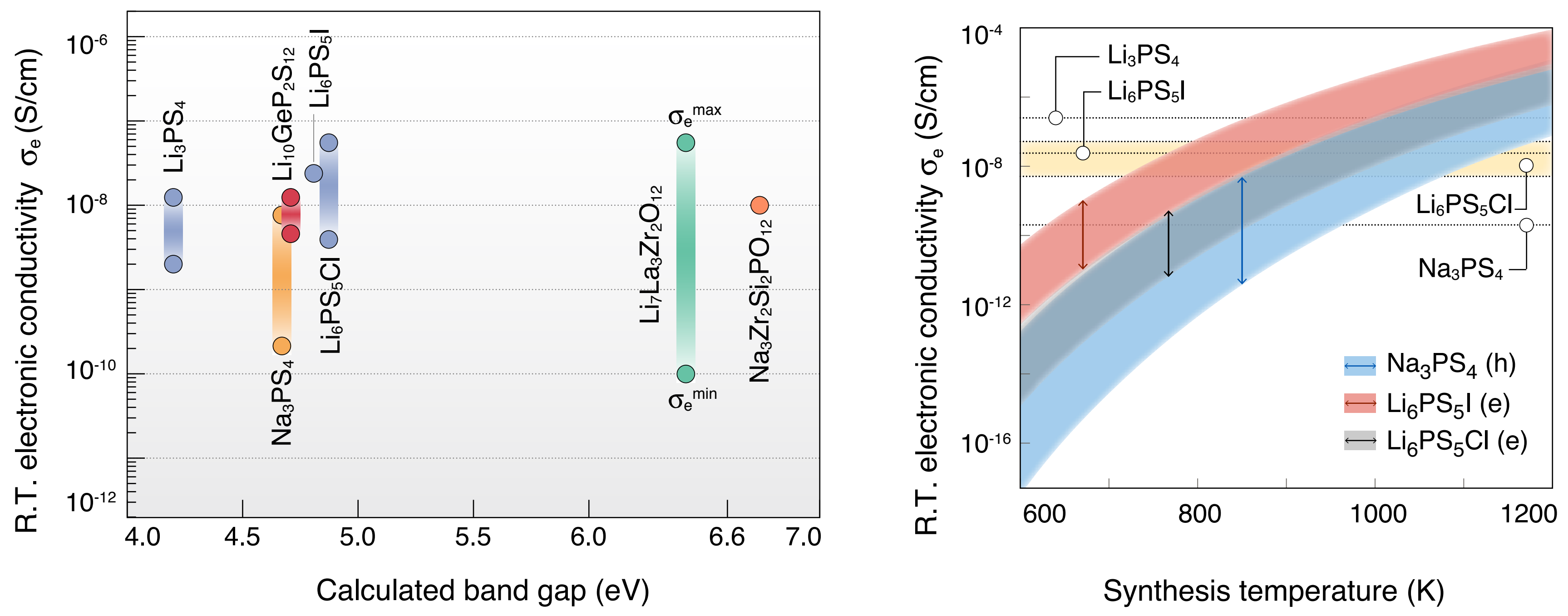


\title{
The Devil is in the Defects: Electronic Conductivity in Solid Electrolytes
}

\author{
Prashun Gorai, ${ }^{a *}$ Theodosios Famprikis, ${ }^{b, c}$ Baltej Singh, ${ }^{d}$ Vladan Stevanović, ${ }^{a *}$ Pieremanuele Canepa ${ }^{d, e, f *}$
}

\begin{abstract}
Rechargeable solid-state batteries continue to gain prominence due to their increased safety. However, a number of outstanding challenges have prevented their adoption in mainstream technology. In this study, we reveal the origins of electronic conductivity $\left(\sigma_{e}\right)$ in solid electrolytes (SEs), which is deemed responsible for solid-state battery degradation, as well as more drastic short-circuit and failure. Using first-principles defect calculations and physics-based models, we predict $\sigma_{e}$ in three topical SEs: $\mathrm{Li}_{6} \mathrm{PS}_{5} \mathrm{Cl}$ and $\mathrm{Li}_{6} \mathrm{PS}_{5} \mathrm{I}$ argyrodites, and $\mathrm{Na}_{3} \mathrm{PS}_{4}$ for post-Li batteries. We treat $\mathrm{SEs}$ as materials with finite band gaps and apply the defect theory of semiconductors to calculate the native defect concentrations and associated electronic conductivities. Our experimental measurements of the band gap of tetragonal $\mathrm{Na}_{3} \mathrm{PS}_{4}$ confirm our predictions. The quantitative agreement of the predicted $\sigma_{e}$ in these three materials and those measured experimentally strongly suggests that self-doping via native defects is the primary source of electronic conductivity in SEs. In particular, we find that $\mathrm{Li}_{6} \mathrm{PS}_{5} X$ are $n$-type (electrons are majority carriers), while $\mathrm{Na}_{3} \mathrm{PS}_{4}$ is $p$-type (holes). Importantly, the predicted values set the lower bound for $\sigma_{e}$ in SEs. We suggest general defect engineering strategies pertaining to synthesis protocols to reduce $\sigma_{e}$ in SEs, and thereby, curtailing the degradation of solid-state batteries. The methodology presented here can be extended to investigate $\sigma_{e}$ in secondary phases that typically form at electrode-electrolyte interfaces, as well as to complex oxide-based SEs.
\end{abstract}

\section{Introduction}

Rechargeable lithium (Li)-ion batteries have revolutionized the industry of portable devices. ${ }^{1-3}$ Concurrently, a daunting task is left to scientists, engineers, and battery manufacturers to develop high-energy density battery architectures that are safe and costs below $150 \$ / \mathrm{kWh}$, which could displace combustion engines in favor of more efficient electric ones. ${ }^{1,3-6}$ The implementation of large-scale Li-ion technologies is challenged by their low safety. ${ }^{7-9}$ Commercial Li-ion batteries contain flammable liquid, non-aqueous organic electrolytes. ${ }^{7,10}$ The dynamic nature of the solid-electrolyte interfaces formed at the electrodes and the high flammability of electrolytes set the conditions, together with external events (e.g., the puncturing of the battery casing), for short circuits, thermal runaways, and possible fires. 7,10,11

Solid-state batteries are considered safer alternatives for energy storage, where liquid electrolytes are replaced by non-flammable solid electrolytes (SEs). ${ }^{22-27}$ Although new SEs display recordhigh $\mathrm{Li}^{+}$-ion conductivities $(>20 \mathrm{mS} / \mathrm{cm})^{21,28-31}$ nearing that of liquid electrolytes (e.g., $1 \mathrm{M} \mathrm{LiPF}_{6}$ in ethylene/dimethyl carbonates $\sim 100 \mathrm{mS} / \mathrm{cm}$ ), ${ }^{25}$ several challenges in solid-state batteries remain unsolved. These are: (i) the formation of highly reactive interfaces between the electrodes and the SE, ${ }^{26,32-39}$ (ii) the loss of physical contact between the electrode and SE over multiple cycles, ${ }^{26,40}$ and (iii) the suppression of dendrites — branched filaments of metallic Li. ${ }^{13,37-39,41-45}$

\footnotetext{
${ }^{a}$ Colorado School of Mines, Golden, CO 80401, USA. ${ }^{b}$ Laboratoire de Réactivité et Chimie des Solides, CNRS UMR 7314, Université de Picardie Jules Verne, 80039 Amiens, France ${ }^{c}$ ALISTORE European Research Institute, CNRS FR 3104, 80039 Amiens, France. ${ }^{d}$ Department of Materials Science and Engineering, National University of Singapore, 9 Engineering Drive 1, Singapore 117575, Singapore. ${ }^{e}$ Chemical and Biomolecular Engineering, National University of Singapore, 4 Engineering Drive 4, Singapore 117585. f ${ }^{f}$ Singapore-MIT Alliance for Research and Technology, 1 CREATE Way, Singapore 138602, Singapore. *E-mail: pgorai@mines.edu,vstevano@mines.edu, pcanepa@nus.edu.sg
}

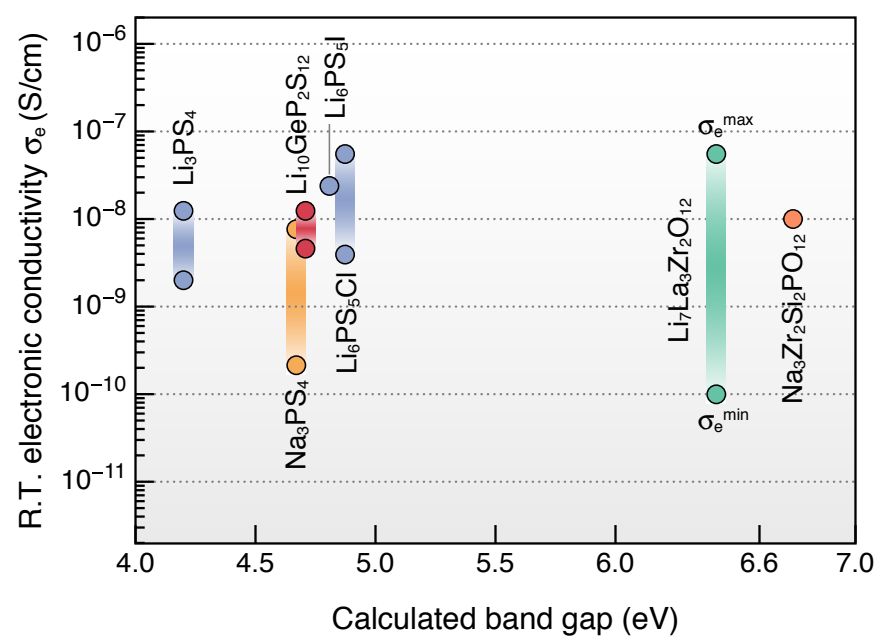

Fig. 1 Plot of experimentally reported room-temperature (R.T.) electronic conductivities $\sigma_{e}$ (in $\mathrm{S} / \mathrm{cm}$ ) of topical SEs vs. their computed band gaps at the GW level of theory. The GW band gap of $\mathrm{Li}_{7} \mathrm{La}_{3} \mathrm{Zr}_{2} \mathrm{O}_{12}$ was taken from Ref. 12. Each bar reports the maximum and minimum values of $\sigma_{e}$ reported in the literature. $\sigma_{e}$ of doped $\mathrm{Li}_{7} \mathrm{La}_{3} \mathrm{Zr}_{2} \mathrm{O}_{12}$ are taken from Ref. 13 (max. value, $\sigma_{e}^{\max }$ for $\mathrm{Li}_{6.4} \mathrm{La}_{3} \mathrm{Zr}_{1.4} \mathrm{Ta}_{0.6} \mathrm{O}_{12}$ ) and from Ref. 14 (min. value, $\sigma_{e}^{\text {min }}$ for $\mathrm{Li}_{6.4} \mathrm{Ga}_{0.2} \mathrm{La}_{3} \mathrm{Zr}_{2.0} \mathrm{O}_{12}$ ). $\mathrm{Na}_{3} \mathrm{Zr}_{2} \mathrm{Si}_{2} \mathrm{PO}_{12}$ was reported by Chen et al. ${ }^{15} \mathrm{Li}_{6} \mathrm{PS}_{5} \mathrm{Cl} \sigma_{e}^{\max }$ from Ref. 16 and $\sigma_{e}^{\text {min }}$ from Ref. 17, and $\mathrm{Li}_{6} \mathrm{PS}_{5} \mathrm{I}$ from Ref. 18. $\mathrm{Li}_{3} \mathrm{PS}_{4} \sigma_{e}^{\max .}$ and $\sigma_{e}^{\min }$ are taken from Refs. 13,19, while $\mathrm{Li}_{10} \mathrm{GeP}_{2} \mathrm{~S}_{12} \sigma_{e}^{\max }$ and $\sigma_{e}^{\min }$ from Refs. 20,21.

The nucleation-growth of Li dendrites in solid-state batteries drives cell failure. ${ }^{33,45}$ Dendrites in SEs and their decomposition products at the interface with electrodes have been linked to an increase in the values of electronic conductivity $\left(\sigma_{e}\right)$ of solid electrolytes. ${ }^{13,26,27}$ Indeed, the electronic conductivity of specific SEs coupled with their facile $\mathrm{Li}^{+}$-ion transport may eventually promote the swift recombination of electrons and $\mathrm{Li}^{+}$ions into metallic $\mathrm{Li}$, resulting in local nucleation of dendrites. ${ }^{13,18,37,41,43-45}$ 
The electron leakage in SEs can also lead to subtle and prolonged processes of battery self-discharge. It has been demonstrated that electrolyte decomposition products at both high and low voltages (vs. $\mathrm{Li} / \mathrm{Li}^{+}$) may display intrinsic electronic conductivity, ${ }^{32,33,38,46}$ whose magnitudes remain elusive. ${ }^{13,14,45}$

While $\sigma_{e}$ values are typically reported for completeness, the focus of many reports (e.g., references in Figure 1) shifts entirely to the high intrinsic $\mathrm{Li}^{+}$-ionic conductivity of novel SE chemistries. Solid electrolytes that are oxides, sulfides, or selenides, and even phosphates and silicates, ${ }^{25}$ typically display band gaps $>4 \mathrm{eV}$ as seen in Figure 1. Thus, the band gap argument i.e., large band gap materials tend to be good electronic insulators, is often used as a descriptor to indicate low electronic conductivities in SEs.

Nevertheless, from Figure 1 it remains impossible to establish any empirical relationship between values of band gaps ( $x$-axis) and respective experimental values of $\sigma_{e}$ (y-axis). If $\sigma_{e}$ were to be proportional to the inverse of the band gap, one would expect large band gap materials, such as $\mathrm{Li}_{7} \mathrm{La}_{3} \mathrm{Zr}_{2} \mathrm{O}_{12}$ (LLZO) or $\mathrm{Na}_{3} \mathrm{Zr}_{2} \mathrm{Si}_{2} \mathrm{PO}_{12}$ to exhibit the lowest electronic conductivities, which is not confirmed by the experimental data in Figure 1. Furthermore, reported values of $\sigma_{e}$ for the same SE, and with the same nominal composition can surprisingly span several orders of magnitude (Figure 1). This variability is ascribed to a number of factors, including different synthesis procedures, different microstructures, different methods to measure $\sigma_{e}$, as well as doping strategies to boost $\mathrm{Li}^{+}$-ion conductivities.

A number of recent studies have investigated the issue of electronic conductivity in SEs. Using neutron depth profiling and other characterization techniques, Han et al., ${ }^{13}$ demonstrated that even ppm-level electronic conductivities can trigger Li dendrite growth. They reported room-temperature $\sigma_{e}$ of $\sim 5.5 \times$ $10^{-8} \mathrm{~S} / \mathrm{cm}$ in LLZO, and $\sim 2.2 \times 10^{-9} \mathrm{~S} / \mathrm{cm}$ in $\mathrm{Li}_{3} \mathrm{PS}_{4}$ and drew the connection to the dendrite growth observed. ${ }^{13}$ Dendrites were not observed in the case of LiPON, ${ }^{13}$ with $\sigma_{e}$ values $\left(10^{-15}-\right.$ $10^{-12} \mathrm{~S} / \mathrm{cm}$ ) at least four orders of magnitude lower than those in LLZO and $\mathrm{Li}_{3} \mathrm{PS}_{4}$. In striking contrast, recently, Philipp et al. ${ }^{14}$ reported $\sigma_{e}$ of $\sim 10^{-10} \mathrm{~S} / \mathrm{cm}$ at $293 \mathrm{~K}$ in single-crystal Ga-doped LLZO, and claimed that such "low" $\sigma_{e}$ cannot be responsible for Li dendrite growth in LLZO. These evidences point to a lack of consensus on the effects of $\sigma_{e}$ in SEs. Notably, there is considerable disparity among the various reports on the acceptable vs. unacceptable values of $\sigma_{e}$. We clarify this aspect in this study.

The emergence of first-principles methods to computationally estimate electronic transport and defect properties offers a practical way to gain insights into and quantify $\sigma_{e}$ in SEs. Here, we adapt a methodology based on density functional theory (DFT) calculations developed in Ref. 47 to estimate carrier (electrons, holes) mobilities. We determine the carrier concentrations and the $\sigma_{e}$ in three topical SEs, i.e., two argyrodites $\mathrm{Li}_{6} \mathrm{PS}_{5} X$ (where $X=\mathrm{Cl}, \mathrm{I}$ ) and $\mathrm{Na}_{3} \mathrm{PS}_{4}$; the latter being important for beyondLi batteries. ${ }^{48-52}$ We identify the most favorable native defects in these SEs that create free carries and give rise to their electronic conductivities. Our results suggest that even SEs with large band gaps may display appreciable electronic conductivities, originating from the existence of charged point defects alone. These predictions of $\sigma_{e}$ set a lower bound for what can be observed experimentally. The presence of extended defects, such as grain boundaries and decomposition phases at the heterogeneous interfaces with the electrodes may also affect $\sigma_{e}$. Facile formation of charged defects at the grain boundary surfaces may create additional trapped charge carriers leading to reduction of $\mathrm{Li}^{+}$ions and in-place nucleation of Li metal. We propose defect engineering strategies to control the synthesis to minimize bulk $\sigma_{e}$.

\section{Point Defects and Electronic Conductivity}

Crystalline solids contain defects at finite temperatures that range from native point defects, extrinsic impurities to grain boundaries. ${ }^{53}$ Atomic-scale point defects are present in metals, semiconductors, and insulators under virtually all conditions. The most common native (intrinsic) point defects include vacancies, anti-sites, and interstitials. For example, in $\mathrm{Li}_{6} \mathrm{PS}_{5} \mathrm{I}$, three types of native point defects can occur and are investigated here: (i) vacancies, e.g. Li vacancy $\left(\mathrm{V}_{\mathrm{Li}}\right)$, (ii) interstitials, e.g. Li interstitial $\left(\mathrm{Li}_{\mathrm{i}}\right)$, and (iii) anti-sites e.g. sulfur on iodine anti-site $\left(\mathrm{S}_{\mathrm{I}}\right)$; symbols in parentheses denote the defects in Kröger-Vink notation. Point defects can be charged (ionized), which may create free carriers - electrons or holes - in the material. Such free carriers will give rise to electronic conductivity. Therefore, to understand and quantify electronic conductivity, one must first determine the thermodynamics of point defect formation. Importantly, here, we treat SEs as materials with finite band gaps. ${ }^{54,55}$ As such, the defect theory developed for semiconductors can be applied to calculate the defect energetics in SEs. ${ }^{55,56}$

\subsection{Defect and Carrier Concentration}

Within the supercell approach, ${ }^{57}$ the formation energy $\Delta E_{\mathrm{D}, \mathrm{q}}$ of a point defect $D$ in charge state $q$ is:

$$
\Delta E_{\mathrm{D}, \mathrm{q}}=\left[E_{\mathrm{D}, \mathrm{q}}-E_{\mathrm{host}}\right]+q E_{\mathrm{F}}+\sum_{i} n_{i} \mu_{\mathrm{i}}+E_{\mathrm{corr}}
$$

where $\left[E_{\mathrm{D}, \mathrm{q}}-E_{\mathrm{host}}\right]$ denotes the total energy difference between the undefected supercell of the SE with no net charge $\left(E_{\text {host }}\right)$ and the supercell with defect $D$ and charge $q$. The term $q E_{\mathrm{F}}$ is the energy of exchanging the charge $q$ with the reservoir of charges described by the Fermi energy $\left(E_{\mathrm{F}}\right) . n_{i}$ is the number of atoms of element $i$ added $\left(n_{i}>0\right)$ or removed $\left(n_{i}<0\right)$ to create the defect $D$. $\mu_{i}$ is the chemical potential of element $i$. Thus, the term $\sum_{i} n_{i} \mu_{i}$ accounts for the energy associated with the exchange of elemental species. Eq. 1 describes the defect formation in the grandcanonical ensemble accounting for exchange of both charge and elemental species with an external reservoir. These terms are calculated from first principles using periodic supercells, which introduces artifacts arising from finite-size effects. Corrections to the formation energy are lumped into $E_{\text {corr }}$ (see Section 6).

For a given SE, one calculates the defect formation energy $\Delta E_{\mathrm{D}, \mathrm{q}}$ for all types of defect of interest in all plausible charge states $(q)$. The results are presented in the form of a "defect diagram"; examples are shown in Figure 3. The $x$-axis is the Fermi energy $\left(E_{\mathrm{F}}\right)$, spanning from the valence band maximum (VBM) to the conduction band minimum (CBM). $E_{\mathrm{F}}$ is conventionally referenced to the VBM, which is set to $0.0 \mathrm{eV}$. Since $\Delta E_{\mathrm{D}, \mathrm{q}}$ is linear 
w.r.t. $E_{\mathrm{F}}$ (Eq. 1), $\Delta E_{\mathrm{D}, \mathrm{q}}$ is a straight line with slope $q$, the charge state of the defect. A donor defect is one with a positive slope (positively charged defect), and an acceptor has negative slope. Charge neutral defects appear as horizontal lines. For a given defect, only the lowest energy charged state at a certain $E_{\mathrm{F}}$ is shown. As such, a change in the slope of the line for a given defect represents the value of $E_{\mathrm{F}}$ where the energetically most favorable charge state changes - this crossover point is the charge transition level. Importantly, the defect formation energy is a function of the elemental chemical potential $\left(\mu_{i}\right)$, which is determined by the chemical or electrochemical environment (Section 3.1).

Under specific synthesis conditions, the equilibrium $E_{\mathrm{F}}$ is set by a charge balance between the charged defects (donors, acceptors) and charge carriers (electrons, holes). A Boltzmann distribution regulates the defect concentration such that $\left[D_{\mathrm{q}}\right]=N_{s} e^{-\Delta E_{\mathrm{D}, \mathrm{q}} / k_{B} T}$, where $\left[D_{\mathrm{q}}\right]$ is the defect concentration, $N_{s}$ is the lattice site concentration where the defect $D$ can be formed, $k_{B}$ is the Boltzmann constant, and $T$ is the temperature. At a given $\mathrm{T}$, the concentration of charge carriers depends only on $E_{\mathrm{F}}$, as per the Fermi-Dirac distribution. When the charges are balanced, the total positive charges equal to the negative charges. The charge neutrality condition can be solved self-consistently to determine the equilibrium $E_{\mathrm{F}}$ and the corresponding defect and carrier concentrations. The net free carrier concentration is $\left|n_{e}-n_{h}\right|$, where $n_{e}$ and $n_{h}$ are electron and hole concentrations. If $n_{e}>n_{h}$, the material is $n$-type and $p$-type when $n_{h}>n_{e}$. Defects formed at the synthesis temperature are assumed to be kinetically "frozen in", i.e. when a material is quenched to lower temperature, e.g. room temperature, the defect concentrations reflect the defect chemistry at the synthesis temperature. Throughout this study we assumed the synthesis temperature to be $800 \mathrm{~K}$, which is a typical synthesis temperature for $\mathrm{Li}_{6} \mathrm{PS}_{5} X$ argyrodites and tetragonal $\mathrm{Na}_{3} \mathrm{PS}_{4} \cdot{ }^{58,59}$

\subsection{Electronic Conductivity}

Within the Drude theory, ${ }^{60}$ the electronic conductivity $\left(\sigma_{e}\right)$ is proportional to the concentration of free charge carriers ( $n$, electrons or holes) and their mobility $(\zeta)$. One can estimate $\sigma_{e}$ by knowing $n$ and $\zeta$ using Eq. 2.

$$
\sigma_{e}=n e \zeta
$$

where $e$ is the electronic charge. Assuming that free carriers arise from the formation of charged point defects in a material, we can estimate $n$ as a function of the synthesis conditions.

The intrinsic, phonon-limited carrier mobility is the upper limit of mobility and can be determined accurately with $a b$ initio methods by computing the electron-phonon coupling matrix, ${ }^{61}$ but such calculation are computationally expensive. To circumvent the direct calculations of $\zeta$, one may adopt a computationally more tractable method. Some of us have previously developed a semi-empirical model to estimate $\zeta$ by fitting measured room-temperature $\zeta$ and parameters calculated from DFT calculations. ${ }^{47}$ Assuming band conduction, the phonon-limited carrier mobility is modeled as:

$$
\zeta=A_{0} B^{S}\left(m_{b}^{*}\right)^{-t}
$$
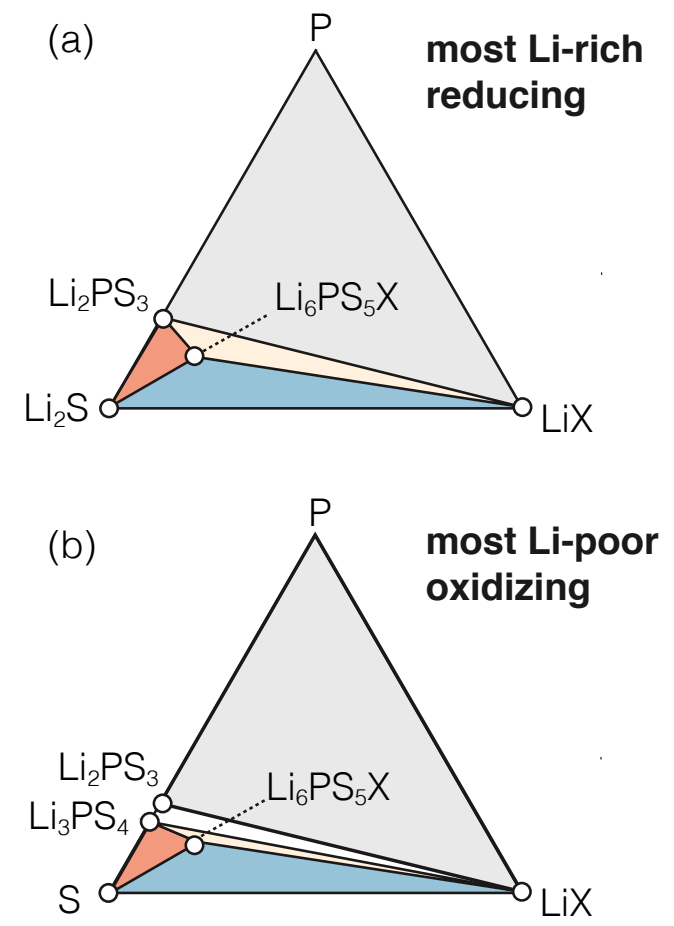

Fig. 2 Projected compound phase diagrams showing the phase equilibria of argyrodite $\mathrm{Li}_{6} \mathrm{PS}_{5} X$ with $X=\mathrm{Cl}$ and $\mathrm{I}$ in the quarternary Li-P-S- $X$ chemical space. Projections are shown for specific Li chemical potentials: (a) most Li-rich or reducing condition, and (b) most Li-poor or oxidizing conditions. Equilibrium tie-lines are drawn between the argyrodite and the neighboring competing phases. The colored triangles set the limits of elemental chemical potentials in Eq. 1.

where $B$ is the bulk modulus, and $m_{b}^{*}$ is the band effective mass of the SE. $A_{0}, s$, and $t$ are constants that were obtained in Ref. 47 by fitting Eq. 3 to experimentally measured room-temperature values of $\zeta$. Therefore, to estimate $\zeta$ using Eq. 3, we compute $B$ and $m_{b}^{*}$, both of which can be calculated accurately with DFT. Details on the calculation of $B$ and $m_{b}^{*}$ are in Section 6 .

\section{Results}

\subsection{Phase Equilibria of $\mathrm{Li}_{6} \mathrm{PS}_{5} \mathrm{I}$}

For a given material, the accessible range of elemental chemical potentials in Eq. 1 is constrained by the condition of its phase stability. The prevailing elemental chemical potential (e.g., $\mu_{\text {element }}$ in Eq. 1) is controlled by the specific synthesis condition of the solid electrolyte and/or the operating electrochemical conditions of the cell as in real experiments.

As an example, we discuss the phase stability of $\mathrm{Li}_{6} \mathrm{PS}_{5} X$ with $X$ $=\mathrm{Cl}$ and $\mathrm{I}$ and the same discussion can be extended to $\mathrm{Na}_{3} \mathrm{PS}_{4}$ in Section S4 of the SI. Figure 2 shows projections of the computed compound quaternary Li-S-P- $X$ phase diagrams at specific electrochemical conditions. Figure 2(a) corresponds to the projection at the most Li-rich condition under which $\mathrm{Li}_{6} \mathrm{PS}_{5} X$ are thermodynamically stable. This also corresponds to thermodynamic stability of $\mathrm{Li}_{6} \mathrm{PS}_{5} X$ in a highly reducing environment. Figure 2(b) shows the phase stability of $\mathrm{Li}_{6} \mathrm{PS}_{5} X$ for the most Li-poor and 
simultaneously, the most S-rich (in this case, equilibrium with elemental S) conditions capturing the stability of $\mathrm{Li}_{6} \mathrm{PS}_{5} X$ at higher voltage. The values of the elemental chemical potentials for the phase stability of $\mathrm{Li}_{6} \mathrm{PS}_{5} \mathrm{I}, \mathrm{Li}_{6} \mathrm{PS}_{5} \mathrm{Cl}$, and $\mathrm{Na}_{3} \mathrm{PS}_{4}$ are listed in Tables S1, S2, and S3 in SI.

The three-phase regions (triangles) formed by the phases in equilibrium with $\mathrm{Li}_{6} \mathrm{PS}_{5} X$ set the accessible range of chemical potentials. Indeed, in Figure 2 (a) $\mathrm{Li}_{6} \mathrm{PS}_{5} X$ is connected by tie lines with a number of compounds, including $\mathrm{Li}_{2} \mathrm{~S}, \mathrm{Li}_{2} \mathrm{PS}_{3}$ and $\mathrm{LiX}$ ( $\mathrm{Cl}$ or I). These findings are in line with previous experimental and theoretical reports. ${ }^{32,33,38,62}$ Notably, Figure 2(a) indicates that $\mathrm{Li}_{6} \mathrm{PS}_{5} \mathrm{I}$ cannot be in direct equilibrium with Li-metal (low voltages); instead, $\mathrm{Li}_{6} \mathrm{PS}_{5} X$ are in equilibrium with the Li-rich phase $\mathrm{Li}_{2} \mathrm{~S}$. Thus, the three-phase region formed by $\mathrm{Li}_{2} \mathrm{~S}, \mathrm{Li}_{2} \mathrm{PS}_{3}$ and $\mathrm{Li}_{6} \mathrm{PS}_{5} X$ is important to study the types of point defects and charge carriers, as well as their concentrations when $\mathrm{Li}_{6} \mathrm{PS}_{5} X$ is subjected to low voltages vs. $\mathrm{Li} / \mathrm{Li}^{+}$. From Figure 2(a), we infer that all argyrodite-type SEs are unstable at low voltage, i.e. $\mathrm{Li} / \mathrm{Li}^{+}-3.04 \mathrm{~V}$ vs. SHE, as also reported previously, ${ }^{32,33,38}$ and accurately captured by our computed phase stability.

At oxidizing conditions, Li-poor conditions, or equivalently higher voltages vs. $\mathrm{Li} / \mathrm{Li}^{+}$, two important stability regions for $\mathrm{Li}_{6} \mathrm{PS}_{5} X$ are identified: $(i)$ the three-phase region formed by $\mathrm{S}$ $\mathrm{Li}_{3} \mathrm{PS}_{4}-\mathrm{Li}_{6} \mathrm{PS}_{5} X$, corresponding to $\mathrm{S}$-rich conditions (equilibrium with elemental S), and (ii) the LiI-S-Li ${ }_{6} \mathrm{PS}_{5} X$, corresponding also to the most I-rich conditions. Notably, the low boiling point of sulfur $\left(\sim 445^{\circ} \mathrm{C}\right)$ may create S-poor conditions during the synthesis of sulfide-based SEs, and consequently, introduce defects that readily form under S-poor conditions. Note that syntheses of sulfide-based SEs are often conducted in S-rich environment, ${ }^{38}$ which justify the investigation of point defects in the S-rich regions of the phase diagram.

A byproduct of plotting the equilibrium phase diagrams, of Figures 2(a) and 2(b), are the thermodynamic stability windows of $\mathrm{Li}_{6} \mathrm{PS}_{5} X$-the voltage range within which $\mathrm{Li}_{6} \mathrm{PS}_{5} X$ are thermodynamically stable. We find that $\mathrm{Li}_{6} \mathrm{PS}_{5} \mathrm{I}$ and $\mathrm{Li}_{6} \mathrm{PS}_{5} \mathrm{Cl}$ have limited stability windows of $\sim 0.278 \mathrm{~V}$ and $0.290 \mathrm{~V}$, in reasonable agreement with previously reported data on the parent compound $\mathrm{Li}_{6} \mathrm{PS}_{5} \mathrm{Cl}(\sim 0.3 \mathrm{~V}) .{ }^{32,33,38}$

\subsection{Native Defect Chemistry of Argyrodites}

We begin by analyzing the energetics of defect formation in argyrodites $\mathrm{Li}_{6} \mathrm{PS}_{5} X(X=\mathrm{I}, \mathrm{Cl})$. In Eq. 1 , the defect formation energy $\left(\Delta E_{\mathrm{D}, \mathrm{q}}\right)$ and consequently, the defect and charge carrier (electron, hole) concentrations are sensitive to the electronic structure of the material, particularly the band gap. We computed the band gaps of $\mathrm{Li}_{6} \mathrm{PS}_{5} X$ using the GW method (see Section 6 for details), which is the state-of-the-art for calculating the electronic structures of semiconductors and insulators, ${ }^{63}$ and GW been previously used in battery research. ${ }^{12,64}$ We find the band gaps of $\mathrm{Li}_{6} \mathrm{PS}_{5} \mathrm{Cl}$ and $\mathrm{Li}_{6} \mathrm{PS}_{5} \mathrm{I}$ are $\sim 4.86$ and $\sim 4.80 \mathrm{eV}$.

The formation energies of native point defects $\left(\Delta E_{\mathrm{D}, \mathrm{q}}\right)$ in $\mathrm{Li}_{6} \mathrm{PS}_{5} \mathrm{I}$ are plotted as a function of the Fermi energy $E_{\mathrm{F}}$ ("defect diagram", see Section 2) in Figure 3. Since $\Delta E_{\mathrm{D}, \mathrm{q}}$ depends on the elemental chemical potentials (Eq. 1), we examine the defect for-

\section{Native Defect Energetics of Argyrodite $\mathrm{Li}_{6} \mathrm{PS}_{5} \mathrm{I}$}
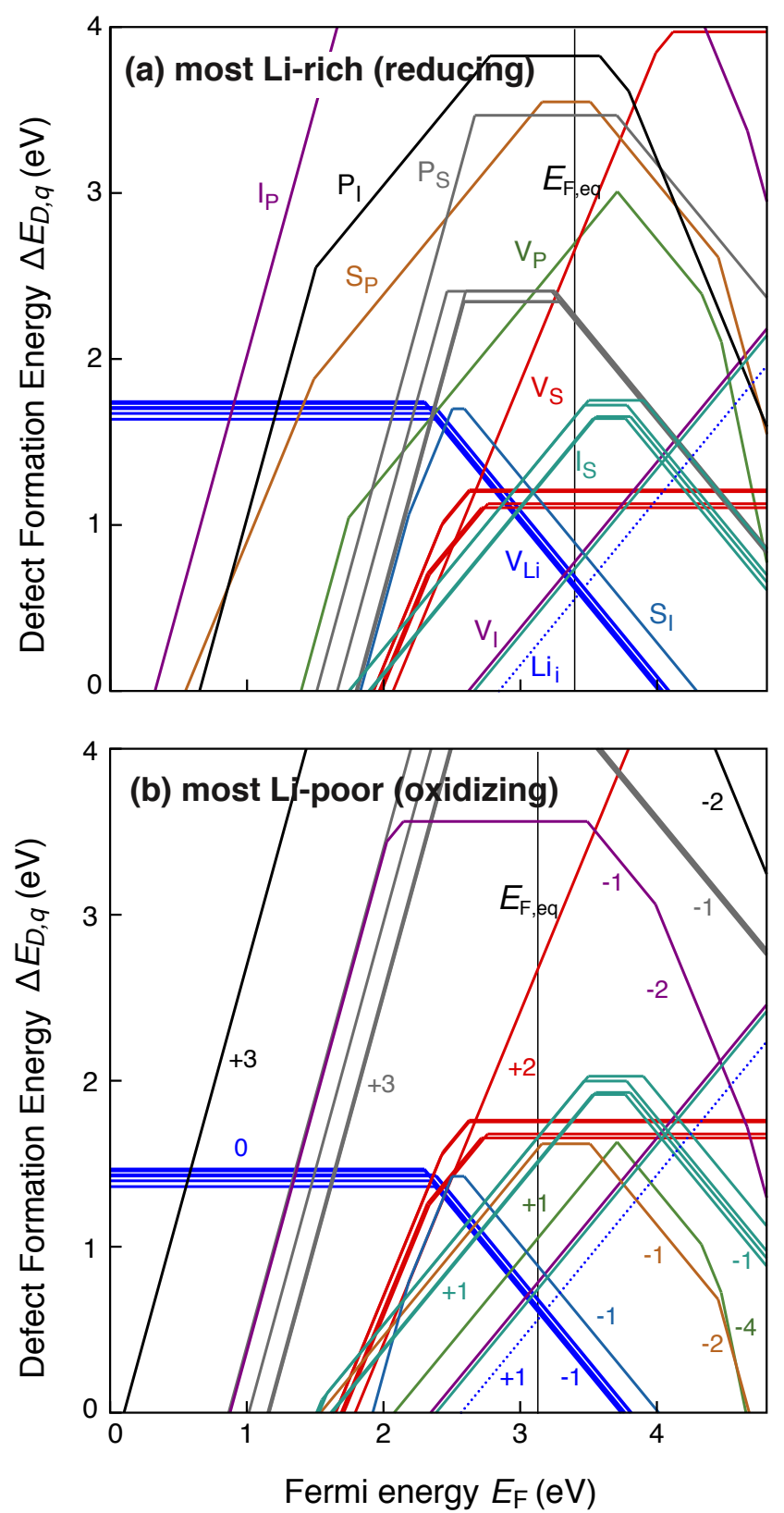

Fig. 3 Formation energies of native point defects $\left(\Delta E_{\mathrm{D}, \mathrm{q}}\right)$ in $\mathrm{Li}_{6} \mathrm{PS}_{5} \mathrm{I}$ as functions of Fermi energy $\left(E_{\mathrm{F}}\right)$ at elemental chemical potentials corresponding to the (a) most Li-rich, and (b) most Li-poor conditions within the phase stability region (see Figure 2 ). $E_{\mathrm{F}}$ is referenced to the valence band maximum. The upper limit of $E_{\mathrm{F}}$ shown is the conduction band minimum such that $E_{\mathrm{F}}$ values range from $0.0 \mathrm{eV}$ to the band gap. Multiple lines of the same color represent the same defect type at different Wyckoff sites. Defects with positive slopes are donors while those with negative slopes are acceptors. The equilibrium Fermi energy $\left(E_{\mathrm{F}, \mathrm{eq}}\right)$, marked by the vertical line, is calculated at $800 \mathrm{~K}$.

mation energetics under the limiting synthesis conditions: most Li-rich or reducing and most Li-poor or oxidizing as discussed in Section 3.1. The dominant defects are the ones with the lowest 
$\Delta E_{\mathrm{D}, \mathrm{q}}$ at the equilibrium Fermi energy, $E_{\mathrm{F}, \mathrm{eq}}$ (black vertical line in Figure 3 ). The slope of each line represents the charge state of the defect as indicated by the numbers next to each line in Figure 3(b) and are color mapped in Figure 3(a). Under both Li-rich (reducing) and Li-poor (oxidizing) conditions, the dominant defects are $\mathrm{Li}$ and $\mathrm{I}$ vacancies $\left(\mathrm{V}_{\mathrm{Li}}, \mathrm{V}_{\mathrm{I}}\right)$, Li interstitials $\left(\mathrm{Li}_{\mathrm{i}}\right)$, and anti-sites $\left(\mathrm{S}_{\mathrm{I}}, \mathrm{I}_{\mathrm{S}}\right)$. Multiple lines of the same color in Figure 3 represent the same defect at different Wyckoff sites, which are symmetry nonequivalent. While the dominant defects are the same under the most Li-rich and Li-poor conditions, their formation energies are different under each condition.

As a SE, it is expected that $\mathrm{Li}$ vacancies and interstitials are among the lowest formation energy defects. Our defect calculations reveal that $\mathrm{Li}_{6} \mathrm{PS}_{5} \mathrm{I}$ also contains appreciable concentrations of iodine vacancies $\left(\mathrm{V}_{\mathrm{I}}\right)$ and sulfur/iodine anti-site defects $\left(\mathrm{S}_{\mathrm{I}}\right.$ and $\mathrm{I}_{\mathrm{S}}$ ). For instance, at a synthesis temperature of $800 \mathrm{~K}$ and under the most Li-rich (reducing) conditions, the concentrations of $V_{I}$ and $\mathrm{I}_{\mathrm{S}}$ are $1.0 \times 10^{17} \mathrm{~cm}^{-3}$ and $5.8 \times 10^{16} \mathrm{~cm}^{-3}$, respectively. In comparison, the concentrations of $\mathrm{Li}$ vacancies and interstitials are $1.5 \times 10^{18} \mathrm{~cm}^{-3}$ and $1.6 \times 10^{18} \mathrm{~cm}^{-3}$, respectively.

The defect energetics of $\mathrm{Li}_{6} \mathrm{PS}_{5} \mathrm{I}$ set the equilibrium Fermi energy $\left(E_{\mathrm{F}, \mathrm{eq}}\right)$ above the mid gap, as indicated by the vertical lines in Figures 3(a) and 3(b). Consequently, $\mathrm{Li}_{6} \mathrm{PS}_{5} \mathrm{I}$ is an $n$-type selfdoped material, with excess free electrons. The free electron concentration is the highest when $\mathrm{Li}_{6} \mathrm{PS}_{5} \mathrm{I}$ is synthesized under the most Li-rich (also, most S-poor) conditions and lowest when grown under the most Li-poor (also, S-rich) conditions. Assuming a typical synthesis temperature of $800 \mathrm{~K}$, the free electron concentration is bounded between $2.0 \times 10^{8} \mathrm{~cm}^{-3}$ (Li-poor) and $1.1 \times 10^{10} \mathrm{~cm}^{-3}$ (Li-rich).

The defect chemistry of $\mathrm{Li}_{6} \mathrm{PS}_{5} \mathrm{Cl}$ (Figure S1) is quantitatively different from that of $\mathrm{Li}_{6} \mathrm{PS}_{5} \mathrm{I}$ - the lowest energy defects are $\mathrm{Li}_{\mathrm{i}}, \mathrm{S}_{\mathrm{Cl}}$, and $\mathrm{Cl}_{\mathrm{S}}$, while $\mathrm{Li}$ and halogen vacancies $\left(\mathrm{V}_{\mathrm{Li}}\right.$ and $\left.\mathrm{V}_{\mathrm{Cl}}\right)$ have comparatively higher formation energies than $\mathrm{Li}_{6} \mathrm{PS}_{5} \mathrm{I}$. Unlike $\mathrm{Li}_{6} \mathrm{PS}_{5} \mathrm{I}$, their analogues sulfur/halogen anti-site defects $\mathrm{S}_{\mathrm{Cl}}$ and $\mathrm{Cl}_{\mathrm{S}}$ in $\mathrm{Li}_{6} \mathrm{PS}_{5} \mathrm{Cl}$ are present in much higher concentrations. At a synthesis temperature of $800 \mathrm{~K}, \mathrm{~S}_{\mathrm{Cl}}$ and $\mathrm{Cl}_{\mathrm{S}}$ concentrations are $2.6 \times 10^{19} \mathrm{~cm}^{-3}$ and $1.9 \times 10^{19} \mathrm{~cm}^{-3}$ under the most Li-rich/Spoor (reducing) synthesis conditions. The sulfur/halogen antisite defect concentrations in $\mathrm{Li}_{6} \mathrm{PS}_{5} \mathrm{Cl}$ are approximately three orders of magnitude larger than in $\mathrm{Li}_{6} \mathrm{PS}_{5} \mathrm{I}$, which is in excellent agreement with the site-disorder observed by Kraft et al. ${ }^{58}$ in $\mathrm{Li}_{6} \mathrm{PS}_{5} \mathrm{Cl}$. The computed $\mathrm{Li}_{\mathrm{i}}$ concentration of $6.6 \times 10^{18} \mathrm{~cm}^{-3}$ is a few times higher than in $\mathrm{Li}_{6} \mathrm{PS}_{5} \mathrm{I}$ under similar synthesis conditions. Furthermore, Kraft et al. ${ }^{58}$ established a direct link between the concentration of $\mathrm{Li}_{i}^{+}$defects and the pre-factor in the Arrhenius expression of the $\mathrm{Li}^{+}$-ion diffusivity, showing that the more defective $\mathrm{Li}_{6} \mathrm{PS}_{5} \mathrm{Cl}$ has a larger pre-factor and higher $\mathrm{Li}^{+}$-ion conductivity than $\mathrm{Li}_{6} \mathrm{PS}_{5} \mathrm{I}$, which is also in excellent agreement with our calculations.

Although the defect concentrations are higher in $\mathrm{Li}_{6} \mathrm{PS}_{5} \mathrm{Cl}$, the concomitant free electron concentrations in $\sim 30$ times lower compared to $\mathrm{Li}_{6} \mathrm{PS}_{5} \mathrm{I}$. At $800 \mathrm{~K}$, the free electron concentrations are bounded between $3.7 \times 10^{8} \mathrm{~cm}^{-3}$ for Li-rich and $6.0 \times 10^{6}$ $\mathrm{cm}^{-3}$ for Li-poor conditions, respectively.

\subsection{Native Defect Chemistry of $\mathrm{Na}_{3} \mathrm{PS}_{4}$}

Next, we examine the defect formation energetics of the tetragonal $\mathrm{Na}_{3} \mathrm{PS}_{4}$, which are shown in the form of defect diagrams in Figure S2. We computed the GW band gap of tetragonal $\mathrm{Na}_{3} \mathrm{PS}_{4}$ to be $\sim 4.67 \mathrm{eV}$.

To asses the quality of the computed band gap we have synthesized and measured the band gap of $\mathrm{Na}_{3} \mathrm{PS}_{4}$ (Section 6.5). Three distinct samples of the $\mathrm{Na}_{3} \mathrm{PS}_{4}$ were investigated: (i) a ball-milled (BM) cubic phase annealed at $260{ }^{\circ} \mathrm{C}$ for $12 \mathrm{~h}$, (ii) a tetragonal phase obtained from the cubic phase after annealing at $500{ }^{\circ} \mathrm{C}$ for $20 \mathrm{~h}$, and (iii) a tetragonal phase as in (ii) but annealed at $500{ }^{\circ} \mathrm{C}$ for 20 minutes. The band gap of $\mathrm{Na}_{3} \mathrm{PS}_{4}$ measured using the Tauc plot of the reflectance data, ${ }^{65}$ modelled with a Kubelka-Munk ${ }^{66}$ function is $\sim 3.9-4 \mathrm{eV}$. The Tauc method is typically used to measure band gaps of semiconductors. ${ }^{65} \mathrm{Al}$ ternatively, the band gap recorded directly from the absorption edge is comparatively larger $\sim 4.0-4.1 \mathrm{eV}$. These values are in qualitative agreement with the large band gap predicted by GW calculations $(\sim 4.67 \mathrm{eV})$. Details on experimental determination of the band gaps are reported in Section S7 of the supplementary information. If the band gap calculated with GW is indeed slightly over-estimated, the carrier concentrations, and therefore, $\sigma_{e}$ are expected to be even higher than our predictions.

The formation energies of native defects under the limiting conditions where $\Delta \mu_{\mathrm{Na}}$ is the highest (reducing) and the lowest (oxidizing) within the phase stability regions of $\mathrm{Na}_{3} \mathrm{PS}_{4}$ are presented in Figure S2 of SI. Under the most Na-rich (most S-poor) synthesis conditions, the lowest-energy defects in $\mathrm{Na}_{3} \mathrm{PS}_{4}$ are $\mathrm{Na}$ vacancy $\left(\mathrm{V}_{\mathrm{Na}}\right)$, Na interstitial $\left(\mathrm{Na}_{\mathrm{i}}\right)$, and $\mathrm{S}$ vacancy $\left(\mathrm{V}_{\mathrm{S}}\right)$, with $\mathrm{V}_{\mathrm{Na}}$ and $\mathrm{Na}_{\mathrm{i}}$ concentrations $\sim 4.06 \times 10^{16} \mathrm{~cm}^{-3}$ at $800 \mathrm{~K}\left(\mathrm{~V}_{\mathrm{Na}}\right.$ concentration slightly higher than $\mathrm{Na}_{\mathrm{i}}$ ). The equilibrium Fermi energies are marked by vertical dotted lines in Figures S2(a) and S2(b) of the SI. In contrast, under the most Na-poor (S-rich, equilibrium with elemental sulfur), the lowest-energy defects are $\mathrm{V}_{\mathrm{Na}}$ and $\mathrm{S}_{\mathrm{Na}}$ antisite defects, such that their concentrations are $\sim 2.9 \times 10^{18} \mathrm{~cm}^{-3}$ at $800 \mathrm{~K}$. Under Na-poor synthesis conditions, $\mathrm{Na}$ interstitial $\left(\mathrm{Na}_{\mathrm{i}}\right)$ concentrations are approximately two orders of magnitude lower $\left(\sim 5.6 \times 10^{14} \mathrm{~cm}^{-3}\right)$ than under Na-rich conditions.

\subsection{Electronic Conductivity of $\mathrm{Li}_{6} \mathrm{PS}_{5} X$ and $\mathrm{Na}_{3} \mathrm{PS}_{4}$}

Here, we estimate the room temperature $(300 \mathrm{~K})$ electronic conductivity of $\mathrm{Li}_{6} \mathrm{PS}_{5} X$ and tetragonal $\mathrm{Na}_{3} \mathrm{PS}_{4}$ due to the formation of charged point defects. To our knowledge, this is the first endeavour to estimate the electronic conductivity in SEs from firstprinciples calculations. The electronic conductivity is a function of the synthesis conditions (specifically, the elemental chemical potentials) and other physical variables, such as the synthesis temperatures. In order, to assess $\sigma_{e}$, we need to estimate the room-temperature carrier mobility $(\zeta)$ using the semi-empirical model of Eq. 3. The computed electron $\left(\zeta_{e}\right)$ and hole $\left(\zeta_{h}\right)$ for each SE are presented in Table 1.

Using the values of $\zeta$ we computed the electronic conductivities at room temperature (Table 1 ). As expected, $\sigma_{e}$ increases with temperature - a direct consequence of the increase in the free or net carrier concentration $\left|n_{e}-n_{h}\right|$. Considering the error of $\pm 1 / 2$ 


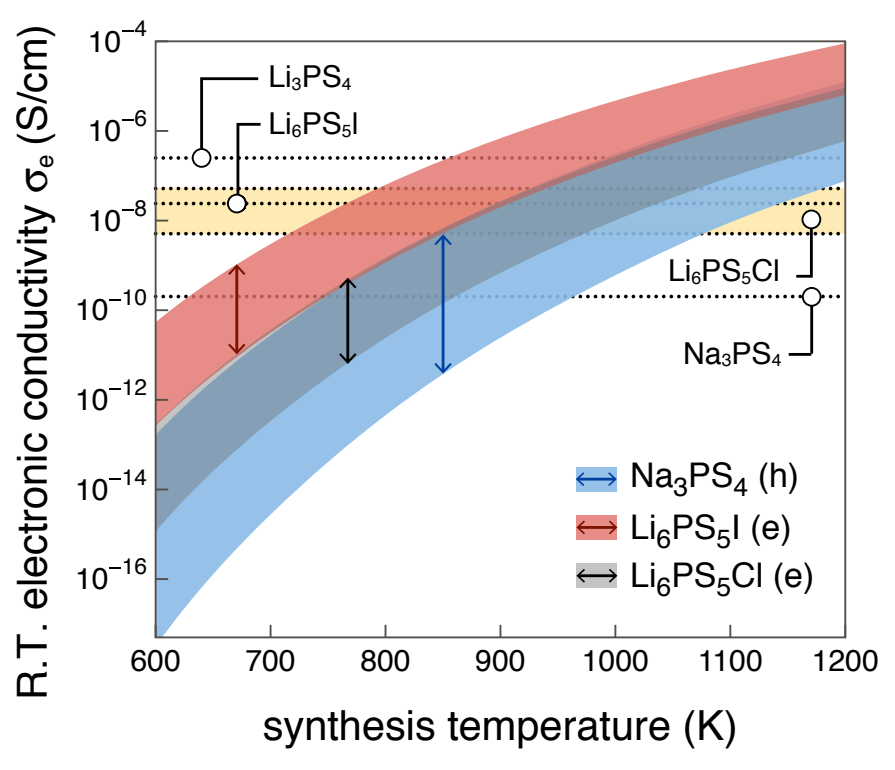

Fig. 4 Computed room temperature (R.T.) $\sigma_{e}$ as a function of the synthesis temperature for three SEs: tetragonal $\mathrm{Na}_{3} \mathrm{P}_{4}$, argyrodites $\mathrm{Li}_{6} \mathrm{PS}_{5} \mathrm{I}$, and $\mathrm{Li}_{6} \mathrm{PS}_{5} \mathrm{Cl}$. The range of $\sigma_{e}$ at any given temperature is set by the range of elemental chemical potentials (synthesis conditions) and denoted by a colored band for each electrolyte. For reference, the experimentally measured electronic conductivities are shown for $\mathrm{Li}_{3} \mathrm{PS}_{4}$ $\left(2.5 \times 10^{-7} \mathrm{~S} / \mathrm{cm}\right),{ }^{13} \mathrm{Li}_{6} \mathrm{PS}_{5} \mathrm{I}\left(2.4 \times 10^{-8} \mathrm{~S} / \mathrm{cm}\right),{ }^{18}$ and $\mathrm{Li}_{6} \mathrm{PS}_{5} \mathrm{Cl}$ $\left(5.1 \times 10^{-9}-5.2 \times 10^{-8} \mathrm{~S} / \mathrm{cm}\right)$ yellow shaded region, ${ }^{16,17}$ and $\mathrm{Na}_{3} \mathrm{PS}_{4}$ $\left(2.1 \times 10^{-10} \mathrm{~S} / \mathrm{cm}\right) .{ }^{67}$

order of magnitude in the estimation of the carrier mobility $(\zeta), 47$ the predicted $\sigma_{\mathrm{e}}$ values are also accurate within $\pm 1 / 2$ order of magnitude (Eq. 2), which is well below the variability observed in the experimental data of Figure 1.

Figure 4 and Table 1 present the computed $\sigma_{e}$ of the three SEs as function of synthesis temperature. Note the majority charge carriers in argyrodites are electrons, while in $\mathrm{Na}_{3} \mathrm{PS}_{4}$, they are holes ( $p$-type). Combined with a high $\zeta_{e}, \mathrm{Li}_{6} \mathrm{PS}_{5} \mathrm{I}$ displays the highest $\sigma_{e}$ among the three SEs (Table 1).

In Table 1 the computed values of $\sigma_{e}$ are either in good agreement or slightly under-estimated compared to the experimental values, which suggests that the experimental measurements of $\sigma_{e}$ include additional sources of charge carriers beyond native point defects. The experimentally measured value by Boulineau et al. ${ }^{18}$ falls within the range of the predicted $\sigma_{e}$. The predicted range of $\sigma_{e}$ for $\mathrm{Li}_{6} \mathrm{PS}_{5} \mathrm{X}(\mathrm{X}=\mathrm{I}, \mathrm{Cl})$ indicates that depending on the synthesis conditions, it can vary by nearly by a factor of 75 , with lower $\sigma_{e}$ values obtained when the argyrodite is synthesized under Lipoor conditions or oxidative environments.

When one compares the lower limits of electronic conductivites between $\mathrm{Li}_{6} \mathrm{PS}_{5} \mathrm{Cl}$ and $\mathrm{Li}_{6} \mathrm{PS}_{5} \mathrm{I}$, the computed $\sigma_{e}$ for $\mathrm{Li}_{6} \mathrm{PS}_{5} \mathrm{Cl}$ is on average $\sim 40$ times lower than $\mathrm{Li}_{6} \mathrm{PS}_{5} \mathrm{I}$ (at $800 \mathrm{~K}$ ). The experimentally reported values, ${ }^{16,17}$ for $\mathrm{Li}_{6} \mathrm{PS}_{5} \mathrm{Cl}\left(\sim 5.1 \times 10^{-9}-5.2 \times 10^{-8}\right.$ $\mathrm{S} / \mathrm{cm}$ ), are shown as yellow shaded region in Figure 4) and slightly higher than the range of computed values.

In the case of $\mathrm{Na}_{3} \mathrm{PS}_{4}$, we find much lower values of $\sigma_{e}$ compared to $\mathrm{Li}_{6} \mathrm{PS}_{5} X$. The reduction of $\sigma_{e}$ in $\mathrm{Na}_{3} \mathrm{PS}_{4}$ originates from a combination of the lower hole mobility $\left(\zeta_{h}\right.$, Table 1)
Table 1 Carrier mobilities (in $\mathrm{cm}^{2} / \mathrm{Vs}$ ) computed with the semi-empirical model (Eq. 3) for the SEs examined. $\zeta_{e}\left(\zeta_{h}\right)$ is the room-temperature, intrinsic electron (hole) mobility. Comparison between the predicted range of room-temperature $\sigma_{e}$ and experimental values of $\sigma_{e}$ (in $\mathrm{S} / \mathrm{cm}$ ).

\begin{tabular}{lcccll}
\hline SE & $\zeta_{\mathbf{e}}$ & $\zeta_{\mathbf{h}}$ & Predicted $\sigma_{\mathbf{e}}$ & \multicolumn{2}{c}{ Experimental $\sigma_{\mathbf{e}}$} \\
\hline $\mathrm{Li}_{6} \mathrm{PS}_{5} \mathrm{I}$ & 35.6 & 1.6 & $0.12-6.28 \times 10^{-8}$ & $2.4 \times 10^{-8}$ & Ref. 18 \\
$\mathrm{Li}_{6} \mathrm{PS}_{5} \mathrm{Cl}$ & 24.9 & 0.7 & $0.02-1.48 \times 10^{-9}$ & $5.1 \times 10^{-9} / 5.2 \times 10^{-8}$ & Ref. 16,17 \\
$\mathrm{Na}_{3} \mathrm{PS}_{4}$ & 9.5 & 2.6 & $0.004-13.1 \times 10^{-10}$ & $2.14 \times 10^{-10}$ & Ref. 67 \\
\hline
\end{tabular}

and lower free hole concentrations, when compared to the argyrodites. Tetragonal $\mathrm{Na}_{3} \mathrm{PS}_{4}$ also shows the largest range of variability among the threes SEs at any given temperature ( $>4$ orders of magnitude assuming synthesis at $\sim 800 \mathrm{~K}$, Figure 4 ).

Notably, this data suggest that in argyrodites-type SEs the lowest value $\sigma_{e}$ is achieved when the material is synthesized under alkali-poor/S-rich conditions, and the highest $\sigma_{e}$ under alkalirich/S-poor conditions. These conditions also correspond to oxidative/high-voltage and reducing/low-voltage conditions, respectively. An inversion in trend is observed for $\mathrm{Na}_{3} \mathrm{PS}_{4}$, where the lowest $\sigma_{e}$ is attained under alkali-rich/S-poor synthesis conditions, comparable to low voltages vs. $\mathrm{Na} / \mathrm{Na}^{+}$.

\section{Discussion}

Previous investigations of native defects in SEs have put emphasis on strategies to improve ionic conductivity. ${ }^{54-56,68,69}$ In this study, we focus on all native defects that may alter the concentration of charge carriers (electrons, holes), in addition to ionic carriers i.e., $\mathrm{Li}^{+}$and $\mathrm{Na}^{+}$ions. In a previous study, ${ }^{55}$ we found $\mathrm{V}_{\mathrm{Na}}^{-}+\mathrm{Li}_{\mathrm{i}}^{+}$Frenkel pairs to be unstable in $\mathrm{Li}_{10} \mathrm{GeP}_{2} \mathrm{~S}_{12}$ such that $\mathrm{Li}_{\mathrm{i}}$ spontaneously relaxes into the neigboring $\mathrm{V}_{\mathrm{Na}}$. By extension, we expect Frenkel pairs to be unstable in similar thiophosphate materials $\mathrm{Li}_{6} \mathrm{PS}_{5} \mathrm{X}$ and $\mathrm{Na}_{3} \mathrm{PS}_{4}$ as well. A deeper understanding of native defects is crucial to rationalize the possible sources of intrinsic electronic conductivities in SEs.

Therefore, we propose a general methodology to estimate the electronic conductivity in bulk SEs. In particular, we have predicted the electronic conductivities of three topical sulfide-based SEs, i.e., $\mathrm{Li}_{6} \mathrm{PS}_{5} \mathrm{I}, \mathrm{Li}_{6} \mathrm{PS}_{5} \mathrm{Cl}$, and $\mathrm{Na}_{3} \mathrm{PS}_{4}$. This choice is justified by the fact that sulfide-based SEs, especially glass-ceramic SEs, seem to be less prone to grain boundary formation. ${ }^{24,27}$ As demonstrated extensively by previous reports, grain boundaries form more readily in oxide-based SEs. ${ }^{24,27,36,39,70-73}$ In particular, grain boundaries might provide an additional source of charge carriers, ${ }^{13}$ and hence, add additional complexity when attempting to systematically understand the crucial role of native points defects in giving rise to electronic conductivity.

\subsection{Influence of Synthesis and Electrochemical Conditions on Native Defect Formation}

The synthesis conditions of solid electrolytes can be tuned to facilitate or curtail the formation of specific types of native defects. The physical and chemical parameters, such as temperature and the elemental chemical potentials are mostly set by the synthesis 
protocols (dry, wet or mechano-synthesis as in ball-milling, etc.), which can greatly influence the formation of specific defects and lead to appreciable variations in the electronic properties of the SE. High-temperature synthesis as well as sintering treatments, such as spark-plasma can not only contribute to significant increase and variation of native point defects but also extended defects. For instance, high-temperature treatments may alter the grain boundary morphology in solid electrolytes and their microstructures.

Notably, different synthesis strategies can be adopted to target the same crystallographic phase of interest, but each strategy may create distinct defects types depending on the synthesis conditions. For example, in this study, the tetragonal $\mathrm{Na}_{3} \mathrm{PS}_{4}$ was produced using both the traditional high-temperature synthesis (at $\sim 1000{ }^{\circ} \mathrm{C}$ ) or alternatively via a rapid thermal annealing at $500{ }^{\circ} \mathrm{C}$ of the ball-milled cubic polymorph (see Section 6). Thus, in computing the dominant native defects, we have carefully considered these aspects. Throughout the discussion, we assumed that the equilibrium temperature (at which defects form) is the typical synthesis temperatures $\sim 800 \mathrm{~K}\left(\sim 527^{\circ} \mathrm{C}\right) .^{16}$

We have identified the important regions of the phase diagram of these solid electrolytes that correspond to actual synthesis conditions. In addition, the phase diagrams are important to study selected electrochemical environments that the SE may experience at specific voltages set by the anode and cathode electrodes (see Figure 2), which provide an understanding of the types of defects that can be formed under an applied bias (as discussed in Section 3.1). An applied voltage is a sufficiently large energy to trigger the decomposition of the SE at the electrodes and form entirely new phases, as noticed in previous experimental and theoretical reports. ${ }^{32,33,38,46,62}$ In addition, simple contact between electrodes and SEs can drive energetic chemical reactions. For example, simply pressing Li-metal against $\mathrm{Li}_{6} \mathrm{PS}_{5} X$ (with $X=\mathrm{Cl}, \mathrm{Br}$ and I), Wenzel et al. could verify the formation of $\mathrm{Li}_{2} \mathrm{~S}, \mathrm{Li} X$ and $\mathrm{Li}_{3} \mathrm{P}$ via X-ray photo-emission spectroscopy. ${ }^{62}$

Aware of the important role the structural disorder can play in influencing the electronic structure and defects properties of $\mathrm{Li}_{6} \mathrm{PS}_{5} \mathrm{I}$ and $\mathrm{Li}_{6} \mathrm{PS}_{5} \mathrm{Cl},{ }^{55,74}$ here, we have undertaken a statistical ensemble approach ${ }^{75}$ to create representative "ordered" structures. Through a sampling of 831 and 1847 structures of $\mathrm{Li}_{6} \mathrm{PS}_{5} \mathrm{I}$ and $\mathrm{Li}_{6} \mathrm{PS}_{5} \mathrm{Cl}$, respectively, which were then relaxed with DFT and their ensemble probabilities calculated (see Section 6), we identified the representative structure that were then utilized for calculating the electronic structure and defect formation energetics. In contrast, tetragonal $\mathrm{Na}_{3} \mathrm{PS}_{4}$ is a fully ordered phase. ${ }^{59}$

Furthermore, the accurate prediction of the nature of specific defects relies on the determination of accurate band gaps in these SEs, which is pivotal to identify the charge carrier concentrations and eventually electronic conductivities. The band gaps were computed using the state-of-the-art GW methodology, which have previously not been reported in the literature. The GW band gaps of $\mathrm{Li}_{6} \mathrm{PS}_{5} \mathrm{X}$ and $\mathrm{Na}_{3} \mathrm{PS}_{4}$ are all $>4.7 \mathrm{eV}$. The GW band gaps are considerably larger than those computed with standard GGAPBE exchange-correlational functional (e.g., $2.27 \mathrm{eV}$ for tetragonal $\left.\mathrm{Na}_{3} \mathrm{PS}_{4}\right)$ and with range-separated HSE06 hybrid functional $(3.49 \mathrm{eV})$, which are frequently adopted as the standard meth- ods in this community. These predictions were confirmed by UV-visible measurements on the tetragonal- $\mathrm{Na}_{3} \mathrm{PS}_{4}$ phase with a band gap in the range 3.9-4.1 eV. Interestingly, the computed band gaps of argyrodites are comparable to that of $\mathrm{Li}_{10} \mathrm{GeP}_{2} \mathrm{~S}_{12}$ $(\sim 4.7 \mathrm{eV}),{ }^{55}$ with both structures containing P-S moieties.

Unsurprisingly, our results suggest that both $\mathrm{Li}$ and Na vacancies as well as their interstitials are among the favorable defects that form in both $\mathrm{Li}_{6} \mathrm{PS}_{5} X$ and $\mathrm{Na}_{3} \mathrm{PS}_{4}$, thereby enabling their high ionic conductivities. ${ }^{16,18,38,56,59,76,77}$ In the case of the $\mathrm{Li}_{6} \mathrm{PS}_{5} X$, we find that the chemical environments, either Li-poor or Li-rich do not alter substantially these defects concentrations. Importantly, these chemical environments can also be accessed at specific electrochemical conditions: a $\mathrm{Li}(\mathrm{Na})$-rich condition represents the situation of the electrolyte placed near a highly reducing materials and mimicked by the low voltage of the $\mathrm{Li}(\mathrm{Na})$ metal electrode (see Figure 2(a)). The electrochemical environment felt by SEs near an high-voltage cathode materials (e.g., voltage $>3.0 \mathrm{~V}$ vs. $\mathrm{Li} / \mathrm{Li}^{+}$) is well captured by the $\mathrm{Li}(\mathrm{Na})$-poor conditions (see Figure 2(b)).

Although $\mathrm{Li}_{6} \mathrm{PS}_{5} X$ is unstable against Li-metal anodes and decomposes into $\mathrm{Li}_{2} \mathrm{~S}, \mathrm{Li}_{3} \mathrm{P}$ and $\mathrm{Li} X$ as in Figure $2,{ }^{62}$ these materials can be safely studied against more positive voltage anodes, e.g. Indium metal $\left(\sim 0.6 \mathrm{~V}\right.$ vs. $\left.\mathrm{Li} / \mathrm{Li}^{+}\right)$. Therefore the Li-rich situations, where the $\mu_{\mathrm{Li}}$ is set by $\mathrm{Li}_{2} \mathrm{~S}, \mathrm{Li}_{3} \mathrm{P}$ and $\mathrm{LiX}$ (Figure 2a) are representative of the environment setup by a higher voltage anodes compared to Li metal.

In striking contrast, $\mathrm{Na}$ vacancies and interstitial defects appear abundant in the $\mathrm{Na}_{3} \mathrm{PS}_{4}$ but only in Na-rich (low voltages vs. $\mathrm{Na} / \mathrm{Na}^{+}$) and sulfur poor conditions. While the high volatility of $\mathrm{S}$ during synthesis conditions may create S-defective electrolytes, on the contrary, our data suggest that $S$ loss during synthesis is beneficial to the formation of $\mathrm{Na}$ vacancies and interstitial that facilitate $\mathrm{Na}^{+}$-ion transport. In the context of this study, we also find that under Na-rich conditions, the electronic conductivity $\left(\sigma_{e}\right)$ is minimized (see Section 3.4). As such, synthesis of $\mathrm{Na}_{3} \mathrm{P}_{4}$ under Na-rich conditions benefits from both higher ionic and lower electronic conductivities.

In both $\mathrm{Li}_{6} \mathrm{PS}_{5} \mathrm{Cl}$ and $\mathrm{Li}_{6} \mathrm{PS}_{5} \mathrm{I}$ anti-site defects, such as $\mathrm{S}_{\mathrm{I}}$ and $\mathrm{I}_{\mathrm{S}}$ as well as $\mathrm{S}_{\mathrm{Cl}}$ and $\mathrm{Cl}_{\mathrm{S}}$ appear favourable. Their concentrations is non-negligible $\left(\sim 10^{16}-10^{19} \mathrm{~cm}^{-3}\right.$ at $\left.\sim 800 \mathrm{~K}\right)$ in both $\mathrm{Li}_{6} \mathrm{PS}_{5} X$ systems. For example, at the nominal synthesis temperature $\sim 500$ ${ }^{\circ} \mathrm{C}(\sim 770-800 \mathrm{~K}),{ }^{58,78}$ of $\mathrm{Li}_{6} \mathrm{PS}_{5} \mathrm{I}$ and at highly reducing conditions (Li-rich), mimicking the environment near the Li-metal anode, we find the concentration of $\mathrm{S}_{\mathrm{I}}$ and $\mathrm{I}_{\mathrm{S}}$ to be in the order $\sim 10^{16} \mathrm{~cm}^{-3}$. The $\mathrm{S}_{\mathrm{Cl}}$ and $\mathrm{Cl}_{\mathrm{S}}$ concentrations are even higher $\sim 10^{19} \mathrm{~cm}^{-3}$. Indeed, our observations are in line with experimental X-ray diffractions on $\mathrm{Li}_{6} \mathrm{PS}_{5} \mathrm{Cl}$, where site disorder on $\mathrm{Cl}$ and $\mathrm{S}$ sites is observed. ${ }^{58,78}$ Recently, Minafra et al. ${ }^{78}$ suggested that the $X / \mathrm{S}$ site disorder is associated with spatially diffuse $\mathrm{Li}^{+}$ distributions, which agrees well with the higher concentrations of $\mathrm{V}_{\mathrm{Li}}$ and $\mathrm{Li}_{\mathrm{i}}$ in $\mathrm{Li}_{6} \mathrm{PS}_{5} \mathrm{Cl}$. In $\mathrm{Na}_{3} \mathrm{PS}_{4}$, anti-site defects $\mathrm{S}_{\mathrm{Na}}$ are expected to be present in higher concentrations under Na-poor conditions.

Therefore, we show that for specific defects there is a strong dependence on the chemical, and especially the electrochemical environment - voltage. However, it remains to be verified ex- 
perimentally whether specific voltages can vary the type of defect locally in the SE, especially at the electrode/electrolyte interfaces. Our model does not capture polarization effects of the electrolyte/electrode interfaces or the effect played by decomposition phases that may form at the electrode. ${ }^{32,33,79}$ Notably, similar predictions can be extended to chart the electronic properties of the decomposition phases forming at the electrode/electrolyte interfaces to infer their intrinsic electronic conductivities and their role in battery degradation.

\subsection{Defect Engineering the Electronic Conductivity of Solid Electrolytes}

The position of the equilibrium Fermi energy $E_{\mathrm{F}, \text { eq }}$ determines the defect and carrier concentrations. In the case of $\mathrm{Li}_{6} \mathrm{PS}_{5} \mathrm{I}$ (Figures $3 \mathrm{a}$ and $3 \mathrm{~b}$ ) the $E_{\mathrm{F}, \mathrm{eq}}$ is pinned between 3.2-3.4 eV with respect to the valence band maximum. $E_{\mathrm{F}, \mathrm{eq}}$ lies in the vicinity of the intersection of $\mathrm{V}_{\mathrm{Li}}^{1-}, \mathrm{Li}_{\mathrm{i}}^{1+}$ and $\mathrm{V}_{\mathrm{I}}^{1+}$. Since $E_{\mathrm{F}, \text { eq }}$ is pinned above the mid gap, $\mathrm{Li}_{6} \mathrm{PS}_{5} \mathrm{I}$ is an $n$-type conductor and the free electron concentration ranges between $10^{8}-10^{10} \mathrm{~cm}^{-3}$ depending on the electrochemical conditions. Likewise, $\mathrm{Li}_{6} \mathrm{PS}_{5} \mathrm{Cl}$ is an $n$-type conductor. While $\mathrm{Li}_{6} \mathrm{PS}_{5} \mathrm{Cl}$ can accommodate larger defect concentrations than $\mathrm{Li}_{6} \mathrm{PS}_{5} \mathrm{I}$, its electron concentration $\left(\sim 10^{6}-10^{8} \mathrm{~cm}^{-3}\right.$ at $\sim 800 \mathrm{~K}$ ) remains $\sim 30$-fold lower than in $\mathrm{Li}_{6} \mathrm{PS}_{5} \mathrm{I}$. These findings are in line with experimental measurements, whereby the $\mathrm{Li}_{6} \mathrm{PS}_{5} \mathrm{I}\left(2.4 \times 10^{-8} \mathrm{~S} / \mathrm{cm}\right)$ posses higher electronic conductivities than $\mathrm{Li}_{6} \mathrm{PS}_{5} \mathrm{Cl}\left(5.1 \times 10^{-9}-5.2 \times 10^{-8} \mathrm{~S} / \mathrm{cm}\right) .{ }^{17,18}$ In contrast, $\mathrm{Na}_{3} \mathrm{PS}_{4}$ appears as a $p$-type self-doped material when synthesized at $\sim 800 \mathrm{~K}$ with predicted hole concentrations $\sim 1.1 \times 10^{6} \mathrm{~cm}^{-3}$ and $3.2 \times 10^{9} \mathrm{~cm}^{-3}$ under Na-rich and Na-poor conditions, respectively. These free charge carrier concentrations may appear negligible, from the perspective of applications such as photovoltaic and thermoelectrics, but as pointed out earlier by He et al., ${ }^{80}$ for solid-state batteries these values may be sufficiently detrimental and contribute subtly to the cell self-discharge.

We estimate the phonon-limited intrinsic carrier mobility $(\zeta)$ using a semi-empirical model. ${ }^{47}$ Typically, $\zeta$ is limited by ionizedimpurity scattering only at lower temperatures when the material is moderately doped, either natively or with extrinsic dopants. For instance, the carrier mobility in Si above temperatures of $\sim 50 \mathrm{~K}$ is dominated by electron-phonon scattering. ${ }^{81}$ Therefore, it is reasonable to assume that $\zeta$ in SEs is limited by phonon scattering. Additional scattering mechanisms, such as disorder scattering may also limit mobility, but it was not explicitly considered in calculating $\zeta$.

With the free carrier concentrations calculated from defect calculations and $\zeta$ calculated with the semi-empirical model, we could then estimate the electronic conductivities of $\mathrm{Li}_{6} \mathrm{PS}_{5} X$ and $\mathrm{Na}_{3} \mathrm{PS}_{4}$ (see Figure 4). The computed band gaps (all $>4.7 \mathrm{eV}$ ) follow the order $\mathrm{Li}_{6} \mathrm{PS}_{5} \mathrm{I}>\mathrm{Li}_{6} \mathrm{PS}_{5} \mathrm{Cl}>$ tetragonal- $\mathrm{Na}_{3} \mathrm{PS}_{4}$ (Figure 4). Given that the electron binding energies in I are lower than in $\mathrm{Cl}$, it is expected to observe $\sigma_{e}\left(\mathrm{Li}_{6} \mathrm{PS}_{5} \mathrm{I}\right)>\sigma_{e}\left(\mathrm{Li}_{6} \mathrm{PS}_{5} \mathrm{Cl}\right)$. Nevertheless, the scarcity of experimental reports of $\sigma_{e}$ warrants future verification of these hypotheses. ${ }^{16-18}$

The electronic conductivity in SEs is routinely measured by two methods: (i) the Hebb-Wagner polarisation, and (ii) DC-

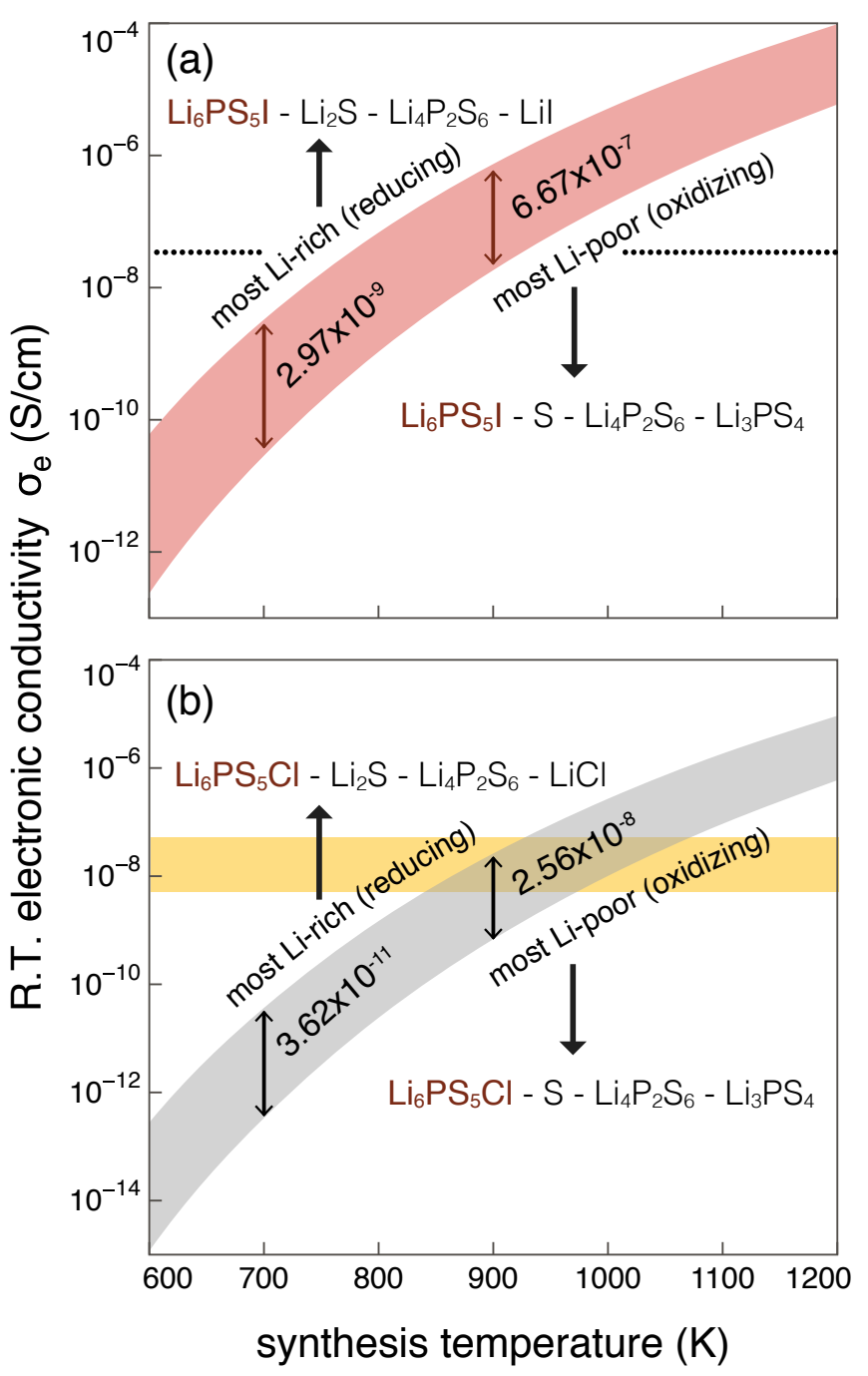

Fig. 5 Predicted range of room temperature (R.T.) electronic conductivity $\left(\sigma_{e}\right)$ in $\mathrm{Li}_{6} \mathrm{PS}_{5} \mathrm{I}$ as function of the synthesis temperature and the chemical environment. The upper bound of $\sigma_{e}$ is set by the Li-rich (reducing) condition in the phase diagram (see Figure $2 \mathrm{a}$ ), whereas the lower bound by the Li-poor or oxidizing conditions. The experimental value of $\sigma_{e}=2.4 \times 10^{-8} \mathrm{~S} / \mathrm{cm}$ is from Ref. 18 . Red arrows are a guide to the eye and indicate the change in electronic conductivity between the upper and lower bound at selected temperatures (700 K and $900 \mathrm{~K}$ ).

polarization. As pointed out by Riess, ${ }^{82}$ the application of the 2-electrode Hebb-Wagner method to mixed ionic-electronic conductors is clearly limited. Instead, a four point Hebb-Wagnertype or van der Pauw setups ensure that the experimental conditions are correct. ${ }^{82}$ Notably, a four point setup is largely utilized in the measurements of carrier mobilities in other energy materials, e.g., solid-oxide fuel cells, thermoelectrics and photovoltaics. ${ }^{83-87}$ Given that our models highlight intrinsic electronic conductivities in SEs, the determinations of $\sigma_{e}$ via DC polarization or Hebb-Wagner measurements remain extremely delicate, especially if the selectivity of the ion or electron-blocking electrodes are not adequate. We urge the community to revisit the current practices in the measurement of $\sigma_{e}$ in SEs and establish rigorous protocols to assess and measure electronic conductivities. 
We now focus on strategies to control the electronic conductivity by defect engineering of $\mathrm{Li}_{6} \mathrm{PS}_{5} \mathrm{X}$ argyrodites. Figure 5 displays the computed and experimental $\sigma_{e}$ as function of synthesis temperature. From Figure 5 the upper bound of $\sigma_{e}$ is set by the Lirich condition, whereas the lower bound by Li-poor regimes. At $\sim 700 \mathrm{~K}$ the upper and lower bounds account for nearly two orders of magnitudes of electronic conductivity. The experimental values of $\sigma_{e}$ (dotted line for $\mathrm{Li}_{6} \mathrm{PS}_{5} \mathrm{I}$ in Figure $5 \mathrm{a}$ and shaded yellow region for $\mathrm{Li}_{6} \mathrm{PS}_{5} \mathrm{Cl}$ in Figure $5 \mathrm{~b}$ ) is qualitatively captured by our predictions. From these observations, we can devise practical and general strategies to reduce $\sigma_{e}$ in SEs:

1. For electron (n-type) conductors, such as the argyrodites, syntheses in Li-poor and oxidizing conditions are advised. In contrast, for hole ( $p$-type) conductors, e.g., $\mathrm{Na}_{3} \mathrm{PS}_{4}$, lower electronic conductivities are achieved under reducing environments (i.e., Na-rich synthesis conditions). While we show that optimal synthesis conditions can be found to minimize electronic conductivity of a specific carrier type, either electrons or holes, these hypotheses should be tested experimentally and perhaps extended to other SEs.

2. The direct link between $\sigma_{e}$ and temperature, clearly suggest that the implementation of soft-chemistry protocols as opposed to typical high-temperature synthesis should be sought. Although mechano-chemical syntheses e.g., via ball milling, are commonly parked under "low temperature methods", the local and instantaneous temperatures experienced by the material range in the order of several hundreds of ${ }^{\circ} \mathrm{C},{ }^{88-90}$ and may even reach $1000{ }^{\circ} \mathrm{C}$. Likewise, sintering procedures for material densification should be cautiously applied. ${ }^{71}$ Note, sintering of solid-electrolytes is commonplace among oxide-based electrolytes including $\mathrm{Li}_{7} \mathrm{La}_{3} \mathrm{Zr}_{2} \mathrm{O}_{12}$ and NaSICONs.

3. Additionally, loss of volatile elements such as S, P etc. from the samples should be carefully controlled. Thus, softchemistry synthesis should be considered. We demonstrated that materials unintentionally grown under S-poor conditions may lead to higher undesired $\sigma_{e}$ e.g. in $\mathrm{Li}_{6} \mathrm{PS}_{5} X$.

4. Controlling the loss of active elements, such as $\mathrm{Li}$ and $\mathrm{Na}$ during syntheses remain more delicate and intimately connected to the nature of charge carriers in specific electrolytes. Specifically, in electron conductors, such as $\mathrm{Li}_{6} \mathrm{PS}_{5} X$ Li-loss will suppress the overall electronic conductivity, where as in $p$-type conductors (e.g., $\mathrm{Na}_{3} \mathrm{PS}_{4}$ ) Na loss will unpleasantly increase $\sigma_{e}$.

5. Therefore, we could envisage employing defect engineering techniques such as phase-boundary mapping, as applied in thermoelectrics, ${ }^{91}$ to precisely control or tune the elemental chemical potentials during synthesis, and consequently, regulate the defect/carrier concentrations, and therefore, $\sigma_{e}$.

6. Another possible strategy to decrease $\sigma_{e}$ involves lowtemperature annealing to re-equilibrate the defects. However, there are challenges with this approach. First, only mobile defects e.g, alkali ions (interstitials) and vacancies may re-equilibrate, whereas the concentrations of defects with significant kinetic barriers for diffusion will remain unchanged. Second, the equilibrium at lower temperatures will lead to lower alkali ion concentrations (following a Boltzmann distribution) and therefore, lower ionic conductivity.

7. We illustrated that the local electrochemical conditions (reducing or oxidizing) are dictated by the electrode potentials, which in turn affect the charge carrier concentrations, and $\sigma_{e}$. Further investigations are needed to elucidate this specific aspect.

These considerations are of general applicability and can be extended to other electrolytes. These design rules may be also suitably modified when developing electrode coating materials, whose specific requirements may be different. ${ }^{92-95}$ It is worth commenting that although accurate band gaps are crucial to identify the equilibrium Fermi energy, and hence, the type and concentrations of electronic charge carriers, the magnitude of the band gap alone cannot be used as a proxy to comment on the severity (or lack thereof) of electronic conductivity in specific SEs.

\section{Conclusions}

A central problem in the development of solid-state batteries is the detrimental intrinsic electronic conductivity that contributes, at best, to the cell self-discharge and at worst, to short circuiting of the battery. In this study, we reveal using first-principles calculations that the native defects are the source of the observed electronic conductivities in the three topical SEs $-\mathrm{Li}_{6} \mathrm{PS}_{5} X$, where $X$ $=\mathrm{Cl}$, I, and tetragonal $\mathrm{Na}_{3} \mathrm{PS}_{4}$. In addition, we quantitatively establish the range of electronic conductivities in these SEs. We leverage this knowledge to propose defect engineering strategies for tuning the defects, and therefore, charge carriers and electronic conductivity. Further work is required to extend the models and the methodologies presented in this study to more complex scenarios such as oxide-based electrolytes, where extended defects (e.g., grain boundaries) may affect electronic conductivity. We urge the battery research community to carefully consider electronic conductivity in SEs. To that end, we believe that rigorous and systematic protocols should be developed to measure electronic conductivity.

\section{Methodology}

\subsection{Structure Selection for Defect Calculation}

In this study, we perform defect calculations on the high-Na ${ }^{+}$ conductivity and fully-ordered polymorph of $\mathrm{Na}_{3} \mathrm{PS}_{4}$, which arranges into a tetragonal crystal structure $\left(P \overline{4} 2_{1} c\right.$, see Figure S3). ${ }^{59}$ A cubic phase of $\mathrm{Na}_{3} \mathrm{PS}_{4}(I \overline{4} 3 m)$ also exist. ${ }^{59}$ The crystal structure of tetragonal $\mathrm{Na}_{3} \mathrm{PS}_{4}$ is composed of $\mathrm{PS}_{4}^{3-}$ polyhedra arranged in a body-centered cubic lattice with two unique $\mathrm{Na}$ Wyckoff sites $(4 d, 2 a)$, and one each for $\mathrm{P}(2 a)$ and $\mathrm{S}(8 e)$.

Unlike $\mathrm{Na}_{3} \mathrm{PS}_{4}$, Li argyrodites $\mathrm{Li}_{6} \mathrm{PS}_{5} \mathrm{X}(\mathrm{X}=\mathrm{Cl}, \mathrm{Br}, \mathrm{I})$ exhibit structural disorder, including partial site occupancy. The site disorder in $\mathrm{Li}_{6} \mathrm{PS}_{5} \mathrm{X}$ was modeled with an ensemble statistical procedure, and as discussed in Section S5 in SI. ${ }^{55}$ A disordered macrostate can be expressed as a thermodynamic average 
of structurally ordered microstates. ${ }^{74,75}$ The identification of the symmetrically distinct orderings in $\mathrm{Li}_{6} \mathrm{PS}_{5} \mathrm{X}$ was performed using the method proposed by Hart and Forcade ${ }^{96}$. We identified 831 and 1847 distinct orderings in the unit cells of $\mathrm{Li}_{6} \mathrm{PS}_{5} X$. For performing defect calculations, we chose the most probable structures marked in Figures S5 and S6 (supplementary information).

\subsection{Calculation of Defect Formation Energy}

First-principles calculations are used to compute the formation energies of native defects as functions of the Fermi energy. We calculate the defect formation energies in $\mathrm{Na}_{3} \mathrm{PS}_{4}$ and $\mathrm{Li}_{6} \mathrm{PS}_{5} X(X$ $=\mathrm{Cl}, \mathrm{I}$ ) using DFT and a standard supercell approach. ${ }^{57}$ Within the supercell approach, the formation energy $\left(\Delta E_{\mathrm{D}, \mathrm{q}}\right)$ of a point defect $D$ in charge state $q$ is calculated as in Eq. 1

In Eq. $1 \mu_{i}$ is chemical potential of element $i$ and $n_{i}$ is the number of atoms of element $i$ added $\left(n_{i}<0\right)$ or removed $\left(n_{i}>0\right)$ from the supercell. $E_{\mathrm{F}}$ is the Fermi energy. $q E_{\mathrm{F}}$ is the characteristic energy of exchanging charge between the defect and the reservoir of charge, the Fermi sea. The supercell approach to predict defect energetics suffers from artifacts due to finite size effects.

Various correction schemes are available to correct for the finite size artefacts and inaccurate electronic structure, and are factored as $E_{\text {corr }}$ in Eq. 1. ${ }^{57}$ Additional artifacts are introduced due to the limitations of DFT, most notably, the underestimation of the band gap with standard functionals, such as in the generalized gradient approximation (GGA) of Perdew-Burke-Ernzerhof (PBE) ${ }^{97}$.

Among the native defects, we considered vacancies, anti-sites, and $\mathrm{Li}$ and $\mathrm{Na}$ interstitials, with each unique Wyckoff site treated as a different defect. For each defect, charge states $q=-3,-2,-1$, $0,1,2,3$ are calculated; for some defects, such as $V_{P}$, additional charge states $q=-5,-4,4,5$ are also calculated. The possible sites for $\mathrm{Li}$ and $\mathrm{Na}$ interstitials are determined by a Voronoi tessellation scheme as in pylada-defects. ${ }^{98}$ In each structure, the energetically most favorable interstitial configuration is assessed by relaxing up to 15-20 different possible interstitial configurations.

The total energies of the supercells are calculated using the GGA-PBE functional within the projector augmented wave (PAW) formalism available in VASP. ${ }^{99}$ The total energies of 128-atom, and 104-atom supercells of $\mathrm{Na}_{3} \mathrm{PS}_{4}$ and $\mathrm{Li}_{6} \mathrm{PS}_{5} X(X=\mathrm{I}$ and $\mathrm{Cl})$, respectively, are calculated with a plane-wave energy cutoff of $340 \mathrm{eV}$ and a $\Gamma$-centered $4 \times 4 \times 4$ Monkhorst pack $k$-point grid to sample the Brillouin zone. The positions of the ions in the defect supercells are relaxed following the procedure of Refs. 91,100.

The elemental chemical potentials $\mu_{i}$ are expressed relative to those of the elements in reference elemental phases as $\mu_{i}=\mu_{i}^{0}+$ $\Delta \mu_{i}$, where $\mu_{i}^{0}$ is the reference chemical potential under standard conditions and $\Delta \mu_{i}$ is the deviation from the reference. $\Delta \mu_{i}=0$ corresponds to $i$-rich conditions. For example, $\Delta \mu_{\mathrm{S}}=0$ (S-rich) corresponds to the equilibrium between $\mathrm{Li}_{6} \mathrm{PS}_{5} \mathrm{I}$ and solid $\mathrm{S}$. The reference chemical potentials $\left(\mu_{i}^{0}\right)$ are fitted to a set of measured formation enthalpies of compounds as in Ref. 101.

$E_{\text {corr }}$ (Eq. 1) is estimated as in Ref. 57,102, and contain: (i) the image charge correction for charged defects, (ii) the potential alignment correction for charged defects, (iii) the band filling correction for shallow defects, and (iv) the correction of the band edges for shallow acceptors/donors. The defect calculations were prepared and analyzed using the pylada-defects software. ${ }^{98}$

The underestimation of the band gap in DFT is remedied by applying individual valence and conduction band edge shifts (relative to the DFT-computed band edges) as determined from GW quasi-particle energies. ${ }^{57,63}$ We used DFT wave functions as input to the GW calculations. The GW eigenvalues are then iterated to self-consistency removing the dependence on the singleparticle energies of the initial DFT calculation. The input DFT wave functions are kept constant during the GW calculations, which allows the interpretation of the GW quasi-particle energies in terms of energy shifts relative to the Kohn-Sham energies. The GW quasi-particle energies are calculated using a $4 \times 4 \times 4$ supercell for $\mathrm{Na}_{3} \mathrm{PS}_{4}$, and $6 \times 4 \times 4$ supercell for $\mathrm{Li}_{6} \mathrm{PS}_{5} \mathrm{I}$ and $\mathrm{Li}_{6} \mathrm{PS}_{5} \mathrm{Cl}$. Under a given synthesis condition, the equilibrium $E_{\mathrm{F}}$ is determined by solving the charge neutrality condition, as discussed in Section 2.1.

\subsection{Estimation of Carrier Mobility}

To estimate the intrinsic carrier mobility $(\zeta)$ using Eq. 3, we need to compute the bulk modulus $(B)$ and the band effective mass $\left(m_{b}^{*}\right)$ of the relevant bands - conduction band for electrons and valence band for holes. $m_{b}^{*}$ is calculated from the density-of-state (DOS) effective mass ( $\left.m_{\text {DOS }}^{*}\right)$ and the band degeneracy $\left(N_{b}\right)$ as:

$$
m_{b}^{*}=N_{b}^{2 / 3} m_{\mathrm{DOS}}^{*}
$$

where we assumed parabolic bands and isotropic transport. The parabolic band approximation is used to extract $m_{\text {DOS }}^{*}$ within a $100 \mathrm{meV}$ energy window from the relevant band edge. $B$ is determined by fitting the Birch-Murnaghan equation of state to a set of volumes and energies calculated with DFT for unit cells isotropically expanded and contracted around the equilibrium volume. The semi-empirical model for estimation of $\zeta$ (Eq. 3) is accurate within half an order of magnitude of experimental values, ${ }^{47}$ which is satisfactory given that even measured $\zeta$ can exhibit orders of magnitude variations depending on the synthesis conditions and sample preparation.

\subsection{Synthesis of $\mathrm{Na}_{3} \mathrm{PS}_{4}$}

The $\mathrm{Na}_{3} \mathrm{PS}_{4}$ samples were synthesized identically to a previous study. ${ }^{77}$ Stoichiometric amounts of $\mathrm{Na}_{2} \mathrm{~S}$ and $\mathrm{P}_{2} \mathrm{~S}_{5}$ (SigmaAldrich) were utilized as received. The high-temperature tetragonal $\mathrm{Na}_{3} \mathrm{PS}_{4}$ phase was synthesized by a solid-state synthesis route. The powder mixture was pelletized and sealed in evacuated carbon-coated quartz tubes. The sample was heated to $500{ }^{\circ} \mathrm{C}$ at $1{ }^{\circ} \mathrm{C} / \mathrm{min}$, held for $20 \mathrm{~h}$ and naturally cooled to room temperature. BM-cubic $\mathrm{Na}_{3} \mathrm{PS}_{4}$ was synthesized by a mechanochemical

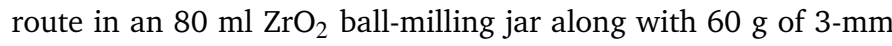
$\mathrm{ZrO}_{2}$ balls. The jars were placed in a planetary ball-mill (Pulverisette 7 Premium, Fritsch) and milled for $12 \mathrm{~h}$ at $500 \mathrm{rpm}$ (144 cycles of 5 minutes of milling followed by 15-minute breaks to dissipate heat). After the milling treatment the resulting powder was subjected to a heat treatment at $260{ }^{\circ} \mathrm{C}$ under vacuum in a vacuum oven (Büchi). BM-tetragonal $\mathrm{Na}_{3} \mathrm{PS}_{4}$ resulted from for 
a rapid thermal annealing of a BM-cubic sample at $500{ }^{\circ} \mathrm{C}$ for 20 minutes. All handling was performed in Ar-filled gloveboxes.

\subsection{Band gap measurements of $\mathrm{Na}_{3} \mathrm{PS}_{4}$}

UV-vis absorption spectra were collected in reflectance mode using a Cary5000 UV-vis-NIR spectrometer (Agilent). $\mathrm{Na}_{3} \mathrm{PS}_{4}$ samples were pressed into $6 \mathrm{~mm}$ diameter pellets and loaded onto a sample holder in the glovebox. To avoid exposure to atmospheric moisture, the samples were sealed behind a glass slide through which the spectra were recorded in the range 250-700 nm using an integrating sphere. The band gap was determined from the Tauc plot of the reflectance data for an indirect allowed transition as presented in the Supporting Information (Section S7 of the Supplementary Information).

\section{Acknowledgement}

P. C. is grateful to the ANR-NRF NRF2019-NRF-ANR073 NaMASTER. P. C. acknowledges funding from the National Research Foundation under his NRF Fellowship NRFF12-2020-0012. P. C. are grateful to the Green Energy program under the project code R284-000-185-731. P. G. acknowledges funding from the Advanced Research Projects Agency Energy (ARPA-E), U.S. Department of Energy, under Award Number DE-AR0001205. The computational work was performed on resources of the National Supercomputing Centre, Singapore (https://www.nscc.sg). The research was partly performed using computational resources sponsored by the Department of Energy's Office of Energy Efficiency and Renewable Energy and located at the National Renewable Energy Laboratory. We are thankful to Prof. Frédéric Sauvage (at LRCS) for help with the UV-Vis spectral measurements and analysis.

\section{References}

1 J.-M. Tarascon, Nat. Chem., 2010, 2, 510-510.

2 B. Dunn, H. Kamath and J.-M. Tarascon, Science, 2011, 334, 928-935.

3 B. Nykvist and M. Nilsson, Nat. Clim. Change., 2015, 5, 329332.

4 D. Larcher and J.-M. Tarascon, Nature Chem, 2015, 7, 1929.

5 Z. P. Cano, D. Banham, S. Ye, A. Hintennach, J. Lu, M. Fowler and Z. Chen, Nature Energy, 2018, 3, 279-289.

6 G. Crabtree, Science, 2019, 366, 422-424.

7 S. S. Zhang, J. Power Sources, 2007, 164, 351-364.

8 H. D. Yoo, E. Markevich, G. Salitra, D. Sharon and D. Aurbach, Mater. Today, 2014, 17, 110-121.

9 K. Liu, Y. Liu, D. Lin, A. Pei and Y. Cui, Sci. Adv., 2018, 4, eaas 9820.

10 J. B. Goodenough and Y. Kim, Chem. Mater., 2010, 22, 587603.

11 E. Peled, J Electrochem Soc, 1979, 126, 2047.

12 T. Thompson, S. Yu, L. Williams, R. D. Schmidt, R. GarciaMendez, J. Wolfenstine, J. L. Allen, E. Kioupakis, D. J. Siegel and J. Sakamoto, ACS Energy Lett., 2017, 2, 462-468.
13 F. Han, A. S. Westover, J. Yue, X. Fan, F. Wang, M. Chi, D. N. Leonard, N. J. Dudney, H. Wang and C. Wang, Nat. Energy, 2019, 4, 187-196.

14 M. Philipp, B. Gadermaier, P. Posch, I. Hanzu, S. Ganschow, M. Meven, D. Rettenwander, G. J. Redhammer and H. M. R. Wilkening, Adv. Mater. Interfaces, 2020, 2000450.

15 S. Chen, C. Wu, L. Shen, C. Zhu, Y. Huang, K. Xi, J. Maier and Y. Yu, Adv. Mater., 2017, 29, 1700431.

16 L. Zhou, K.-H. Park, X. Sun, F. Lalère, T. Adermann, P. Hartmann and L. F. Nazar, ACS Energy Letters, 2018, 4, 265-270.

17 H.-J. Deiseroth, J. Maier, K. Weichert, V. Nickel, S.-T. Kong and C. Reiner, Z. Anorg. Allg. Chem., 2011, 637, 1287-1294.

18 S. Boulineau, M. Courty, J.-M. Tarascon and V. Viallet, Solid State Ionics, 2012, 221, 1-5.

19 K. Minami, F. Mizuno, A. Hayashi and M. Tatsumisago, Solid State Ionics, 2007, 178, 837-841.

20 B. R. Shin, Y. J. Nam, D. Y. Oh, D. H. Kim, J. W. Kim and Y. S. Jung, Electrochim. Acta, 2014, 146, 395-402.

21 N. Kamaya, K. Homma, Y. Yamakawa, M. Hirayama, R. Kanno, M. Yonemura, T. Kamiyama, Y. Kato, S. Hama, K. Kawamoto and A. Mitsui, Nat. Mater., 2011, 10, 682-686.

22 R. P. Rao and S. Adams, Phys. Status Solidi A, 2011, 208, 1804-1807.

23 M. Tatsumisago and A. Hayashi, Solid State Ionics, 2012, 225, 342-345.

24 M. Tatsumisago, M. Nagao and A. Hayashi, J. Asian Ceram. Soc., 2013, 1, 17-25.

25 J. C. Bachman, S. Muy, A. Grimaud, H.-H. Chang, N. Pour, S. F. Lux, O. Paschos, F. Maglia, S. Lupart, P. Lamp, L. Giordano and Y. Shao-Horn, Chem. Rev., 2015, 116, 140-162.

26 J. Janek and W. G. Zeier, Nat Energy, 2016, 1, 16141.

27 T. Famprikis, P. Canepa, J. A. Dawson, M. S. Islam and C. Masquelier, Nat. Mater., 2019, 18, 1278-1291.

28 P. R. Rayavarapu, N. Sharma, V. K. Peterson and S. Adams, J. Solid State Electrochem., 2011, 16, 1807-1813.

29 S. Adams and R. P. Rao, J. Mater. Chem., 2012, 22, 7687.

30 Y. Kato, S. Hori, T. Saito, K. Suzuki, M. Hirayama, A. Mitsui, M. Yonemura, H. Iba and R. Kanno, Nature Energy, 2016, 1, 16030.

31 Y. Gao, A. M. Nolan, P. Du, Y. Wu, C. Yang, Q. Chen, Y. Mo and S.-H. Bo, Chem. Rev., 2020, DOI: 10.1021/acs.chemrev.9b00747.

32 Y. Zhu, X. He and Y. Mo, ACS Appl. Mater. Interfaces, 2015, 7, 23685-23693.

33 W. D. Richards, L. J. Miara, Y. Wang, J. C. Kim and G. Ceder, Chem. Mater., 2015, 28, 266-273.

34 J. Auvergniot, A. Cassel, J.-B. Ledeuil, V. Viallet, V. Seznec and R. Dedryvère, Chem. Mater., 2017, 29, 3883-3890.

35 R. Koerver, W. Zhang, L. de Biasi, S. Schweidler, A. O. Kondrakov, S. Kolling, T. Brezesinski, P. Hartmann, W. G. Zeier and J. Janek, Ener. Environ. Sci., 2018, 11, 2142-2158.

36 K. T. Butler, G. S. Gautam and P. Canepa, npj Comput. Mater., 2019, 5, 19.

37 J. Kasemchainan, S. Zekoll, D. S. Jolly, Z. Ning, G. O. Hart- 
ley, J. Marrow and P. G. Bruce, Nature Materials, 2019, 18, 1105-1111.

38 T. K. Schwietert, V. A. Arszelewska, C. Wang, C. Yu, A. Vasileiadis, N. J. J. de Klerk, J. Hageman, T. Hupfer, I. Kerkamm, Y. Xu, E. van der Maas, E. M. Kelder, S. Ganapathy and M. Wagemaker, Nature Materials, 2020, 19, 428435.

39 Z. Deng, G. S. Gautam, S. K. Kolli, J.-N. Chotard, A. K. Cheetham, C. Masquelier and P. Canepa, Chem. Mater., 2020, 32, 7908-7920.

40 C. Yu, S. Ganapathy, E. R. H. van Eck, H. Wang, S. Basak, Z. Li and M. Wagemaker, Nat. Commun., 2017, 8, 1086.

41 L. Porz, T. Swamy, B. W. Sheldon, D. Rettenwander, T. Frömling, H. L. Thaman, S. Berendts, R. Uecker, W. C. Carter and Y.-M. Chiang, Adv. Energy Mater., 2017, 7, 1701003.

42 E. J. Cheng, A. Sharafi and J. Sakamoto, Electrochim. Acta, 2017, 223, 85-91.

43 L. E. Marbella, S. Zekoll, J. Kasemchainan, S. P. Emge, P. G. Bruce and C. P. Grey, Chem. Mater., 2019, 31, 2762-2769.

44 Y. Song, L. Yang, L. Tao, Q. Zhao, Z. Wang, Y. Cui, H. Liu, Y. Lin and F. Pan, J. Mater. Chem.A, 2019, 7, 22898-22902.

45 T. Krauskopf, F. H. Richter, W. G. Zeier and J. Janek, Chem. Rev., 2020, 120, 7745-7794.

46 S. Wenzel, S. Randau, T. Leichtweiß, D. A. Weber, J. Sann, W. G. Zeier and J. Janek, Chem. Mater., 2016, 28, 24002407.

47 J. Yan, P. Gorai, B. Ortiz, S. Miller, S. A. Barnett, T. Mason, V. Stevanović and E. S. Toberer, Energy Environ. Sci., 2015, 8, 983-994.

48 S. P. Ong, V. L. Chevrier, G. Hautier, A. Jain, C. Moore, S. Kim, X. Ma and G. Ceder, Ener. Environ. Sci., 2011, 4, 3680 .

49 S.-W. Kim, D.-H. Seo, X. Ma, G. Ceder and K. Kang, Adv. Energy Mater., 2012, 2, 710-721.

50 C. Masquelier and L. Croguennec, Chem. Rev., 2013, 113, 6552-6591.

51 N. Yabuuchi, K. Kubota, M. Dahbi and S. Komaba, Chem. Rev., 2014, 114, 11636-11682.

52 I.-H. Chu, C. S. Kompella, H. Nguyen, Z. Zhu, S. Hy, Z. Deng, Y. S. Meng and S. P. Ong, Sci. Rep., 2016, 6, 33733.

53 M. D. McCluskey and E. E. Haller, Dopants and Defects in Semiconductors, CRC Press, 2018.

54 P. Canepa, G. S. Gautam, D. Broberg, S.-H. Bo and G. Ceder, Chem. Mater., 2017, 29, 9657-9667.

55 P. Gorai, H. Long, E. Jones, S. Santhanagopalan and V. Stevanović, J. Mater. Chem. A, 2020, 8, 3851-3858.

56 K. Oh, D. Chang, B. Lee, D.-H. Kim, G. Yoon, I. Park, B. Kim and K. Kang, Chem. Mater., 2018, 30, 4995-5004.

57 S. Lany and A. Zunger, Phys. Rev. B, 2008, 78, 235104.

58 M. A. Kraft, S. P. Culver, M. Calderon, F. Böcher, T. Krauskopf, A. Senyshyn, C. Dietrich, A. Zevalkink, J. Janek and W. G. Zeier, J. Am. Chem. Soc., 2017, 139, 1090910918.

59 T. Krauskopf, S. P. Culver and W. G. Zeier, Inorganic Chem- istry, 2018, 57, 4739-4744.

60 N. W. Ashcroft, N. D. Mermin et al., Solid State Physics, (Harcourt College Publishers Orlando,USA, 1976.

61 F. Giustino, Rev. Mod. Phys., 2017, 89, 015003.

62 S. Wenzel, S. J. Sedlmaier, C. Dietrich, W. G. Zeier and J. Janek, Solid State Ionics, 2018, 318, 102-112.

63 H. Peng, D. O. Scanlon, V. Stevanovic, J. Vidal, G. W. Watson and S. Lany, Phys. Rev. B, 2013, 88, 115201.

64 D.-H. Seo, A. Urban and G. Ceder, Phys. Rev. B, 2015, 92, 115118.

65 J. Tauc, Mater. Res. Bull., 1968, 3, 37-46.

66 W. E. Vargas and G. A. Niklasson, Appl. Opt., 1997, 36, 5580.

67 H. Nguyen, A. Banerjee, X. Wang, D. Tan, E. A. Wu, J.M. Doux, R. Stephens, G. Verbist and Y. S. Meng, J. Power Sources, 2019, 435, 126623.

68 S. Mukhopadhyay, T. Thompson, J. Sakamoto, A. Huq, J. Wolfenstine, J. L. Allen, N. Bernstein, D. A. Stewart and M. D. Johannes, Chem. Mater., 2015, 27, 3658-3665.

69 A. G. Squires, D. O. Scanlon and B. J. Morgan, Chem. Mater., 2019, 32, 1876-1886.

70 A. Sharafi, C. G. Haslam, R. D. Kerns, J. Wolfenstine and J. Sakamoto, J. Mater. Chem. A, 2017, 5, 21491-21504.

71 P. Canepa, J. A. Dawson, G. S. Gautam, J. M. Statham, S. C. Parker and M. S. Islam, Chem. Mater., 2018, 30, 3019-3027.

72 J. A. Dawson, P. Canepa, T. Famprikis, C. Masquelier and M. S. Islam, J. Am. Chem. Soc., 2018, 140, 362-368.

73 J. A. Dawson, P. Canepa, M. J. Clarke, T. Famprikis, D. Ghosh and M. S. Islam, Chem. Mater., 2019, 31, 5296-5304.

74 R. F. Moran, D. McKay, P. C. Tornstrom, A. Aziz, A. Fernandes, R. Grau-Crespo and S. E. Ashbrook, J. Amer. Chem. Soc., 2019, 141, 17838.

75 E. Jones and V. Stevanovic, npJ Computational Materials, 2020, 6, 56.

76 Z. Zhang, Y. Shao, B. Lotsch, Y.-S. Hu, H. Li, J. Janek, L. F. Nazar, C.-W. Nan, J. Maier, M. Armand and L. Chen, Energy Environ. Sci., 2018, 11, 1945-1976.

77 T. Famprikis, O. U. Kudu, J. Dawson, P. Canepa, F. Fauth, E. Suard, M. Zbiri, D. Dambournet, O. Borkiewicz, H. Bouyanfif, S. Emge, C. P. Grey, S. Cretu, J.-N. Chotard, W. Zeier, S. Islam and C. Masquelier, J. Am. Chem. Soc., 2020, 142, 18422-18436.

78 N. Minafra, M. A. Kraft, T. Bernges, C. Li, R. Schlem, B. J. Morgan and W. G. Zeier, Inorg. Chem., 2020, 59, 1100911019.

79 S. Stegmaier, J. Voss, K. Reuter and A. C. Luntz, Chem. Mater., 2017, 29, 4330-4340.

80 X. He, Y. Zhu and Y. Mo, Nat. Commun., 2017, 8, 15893.

81 M. Cardona and Y. Y. Peter, Fundamentals of Semiconductors, Springer, 2005.

82 I. Riess, Solid State Ionics, 1996, 91, 221-232.

83 I. Riess, Solid State Ion., 1982, 7, 307-315.

84 K. A. Borup, J. de Boor, H. Wang, F. Drymiotis, F. Gascoin, X. Shi, L. Chen, M. I. Fedorov, E. Müller, B. B. Iversen and G. J. Snyder, Ener. Environ. Sci., 2015, 8, 423-435. 
85 P. Kumar, D. V. M. Repaka and K. Hippalgaonkar, Rev. Sci. Instrum., 2017, 88, 125112.

86 A. Zevalkink, D. M. Smiadak, J. L. Blackburn, A. J. Ferguson, M. L. Chabinyc, O. Delaire, J. Wang, K. Kovnir, J. Martin, L. T. Schelhas, T. D. Sparks, S. D. Kang, M. T. Dylla, G. J. Snyder, B. R. Ortiz and E. S. Toberer, Applied Physics Reviews, 2018, 5, 021303.

87 T.-R. Wei, M. Guan, J. Yu, T. Zhu, L. Chen and X. Shi, Joule, 2018, 2, 2183-2188.

88 M. L. Trudeau, R. Schulz, D. Dussault and A. V. Neste, Phys. Rev. Lett., 1990, 64, 99-102.

89 C. Koch, Nanostruct. Mater., 1997, 9, 13-22.

90 V. Šepelák, S. Bégin-Colin and G. L. Caër, Dalton Trans., 2012, 41, 11927.

91 S. Ohno, K. Imasato, S. Anand, H. Tamaki, S. D. Kang, P. Gorai, H. K. Sato, E. S. Toberer, T. Kanno and G. J. Snyder, Joule, 2017, 2, 141.

92 M. Aykol, S. Kim, V. I. Hegde, D. Snydacker, Z. Lu, S. Hao, S. Kirklin, D. Morgan and C. Wolverton, Nat. Commun., 2016, 7, 13779.

93 Y. Xiao, Y. Wang, S.-H. Bo, J. C. Kim, L. J. Miara and
G. Ceder, Nat. Rev. Mater., 2019, 5, 105-126.

94 Y. Xiao, L. J. Miara, Y. Wang and G. Ceder, Joule, 2019, 3, 1252-1275.

95 S. P. Culver, R. Koerver, W. G. Zeier and J. Janek, Adv. Energy Mater., 2019, 9, 1900626.

96 G. L. W. Hart and R. W. Forcade, Phys. Rev. B, 2008, 77, 224115.

97 J. P. Perdew, K. Burke and M. Ernzerhof, Phys. Rev. Lett., 1996, 77, 3865.

98 A. Goyal, P. Gorai, H. Peng, S. Lany and V. S. Stevanović, Comp. Mat. Sci., 2016, 130, 1.

99 G. Kresse and J. Furthmüller, Phys. Rev. B, 1996, 54, 11169.

100 P. Gorai, B. Ortiz, E. S. Toberer and V. Stevanovic, J. Mater. Chem. A, 2018, 6, 13806.

101 V. Stevanović, S. Lany, X. Zhang and A. Zunger, Phys. Rev. B, 2012, 85, 115104.

102 C. Freysoldt, B. Grabowski, T. Hickel, J. Neugebauer, G. Kresse, A. Janotti and C. G. V. de Walle, Rev. Mod. Phys., 2014, 86, 253-305. 


\title{
- Electronic Supplementary Information
}

\section{“The Devil is in the Defects: Electronic Conductivity in Solid Electrolytes"}

\author{
Prashun Gorai, ${ }^{*, \dagger}$ Theodosios Famprikis, ${ }^{\ddagger}$ Gill Baltej Singh, ${ }^{\S}$ Vladan Stevanović, ${ }^{* \dagger}$ and

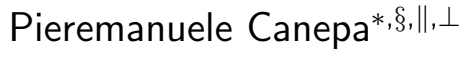 \\ $\dagger$ Colorado School of Mines, Golden, CO, USA. \\ $\ddagger$ Laboratoire de Réactivité et Chimie des Solides, CNRS UMR 7314, Université de Picardie Jules Verne, \\ 80039 Amiens, France \\ \ALISTORE European Research Institute, CNRS FR 3104, 80039 Amiens, France. \\ $\S$ National University of Singapore, 9 Engineering Drive 1, Singapore 117575, Singapore. \\ ||Chemical and Biomolecular Engineering, National University of Singapore, 4 Engineering Drive 4, \\ Singapore 117585. \\ $\perp$ Singapore-MIT Alliance for Research and Technology, 1 CREATE Way, Singapore 138602, Singapore.
}

E-mail: pgorai@mines.edu; vstevano@mines.edu; pcanepa@nus.edu.sg 


\section{Contents}

S1 Formation Energetics of Native Defects in Argyrodite $\mathbf{L i}_{6} \mathbf{P S}_{5} \mathrm{Cl}$

S2 Formation Energetics of Native Defects in Tetragonal $\mathrm{Na}_{3} \mathrm{PS}_{4} \quad 4$

S3 Phase Stability in Chemical Potential Space 5

$\begin{array}{ll}\text { S4 Phase Equilibria of } \mathrm{Li}_{6} \mathrm{PS}_{5} \mathrm{Cl} \text { and } \mathrm{Na}_{3} \mathrm{PS}_{4} & 7\end{array}$

$\begin{array}{lr}\text { S5 Ensemble Statistics for Modeling Disorder in Argyrodites } & 8\end{array}$

S6 Crystal Structure of Tetragonal $\mathrm{Na}_{3} \mathrm{PS}_{4} \quad 10$

S7 Band-gap Measurements of the Tetragonal and Cubic $\mathrm{Na}_{3} \mathrm{PS}_{4}$ Phases $\quad 11$

$\begin{array}{ll}\text { References } & 14\end{array}$ 


\section{S1 Formation Energetics of Native Defects in Argyrodite $\mathrm{Li}_{6} \mathrm{PS}_{5} \mathrm{Cl}$}

The computed formation energies of native point defects, $\Delta E_{D, q}$ in $\mathrm{Li}_{6} \mathrm{PS}_{5} \mathrm{Cl}$ are plotted as a function of the Fermi energy $E_{\mathrm{F}}$ ("defect diagram", see Methodology Section in the main text) in Figure S1. Since $\Delta E_{D, q}$ depends on the elemental chemical potentials (Eq. 1 in the main text), we examine the defect formation energetics under the limiting growth conditions: most Li-rich or reducing and most Li-poor or oxidizing as shown in Figure S3.
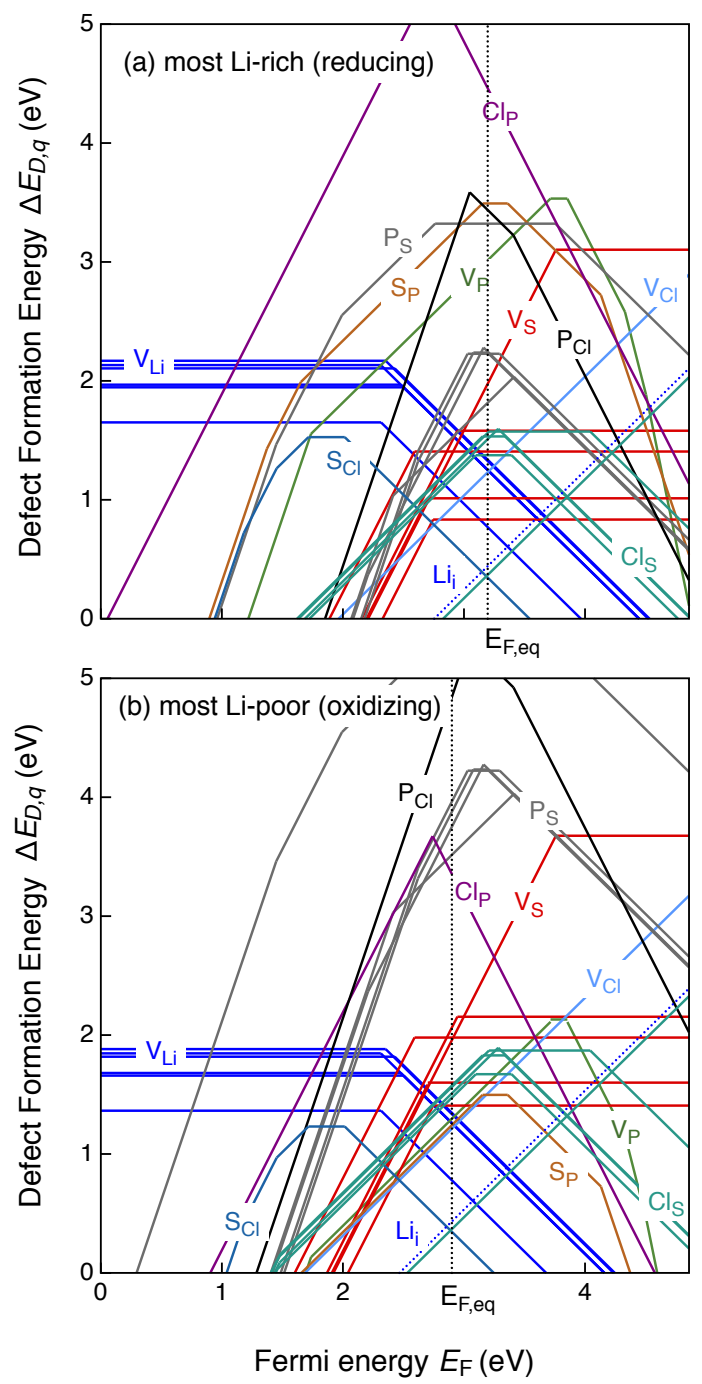

Figure S1: Formation energies of native point defects, $\Delta E_{D, q}$ in $\mathrm{Li}_{6} \mathrm{PS}_{5} \mathrm{Cl}$ as functions of Fermi energy $\left(E_{\mathrm{F}}\right)$ at elemental chemical potentials corresponding to the (a) most Li-rich, and (b) most Li-poor conditions within the phase stability region (see Table S1). $E_{\mathrm{F}}$ is referenced to the valence band maximum. The upper limit of $E_{\mathrm{F}}$ shown is the conduction band minimum such that $E_{\mathrm{F}}$ values range from 0 to the band gap. Multiple lines of the same color represent the same defect type at different Wyckoff sites. The equilibrium Fermi energy $\left(E_{\mathrm{F}, \text { eq }}\right)$, marked by the vertical line, is calculated at $800 \mathrm{~K}$. 


\section{S2 Formation Energetics of Native Defects in Tetragonal $\mathrm{Na}_{3} \mathrm{PS}_{4}$}
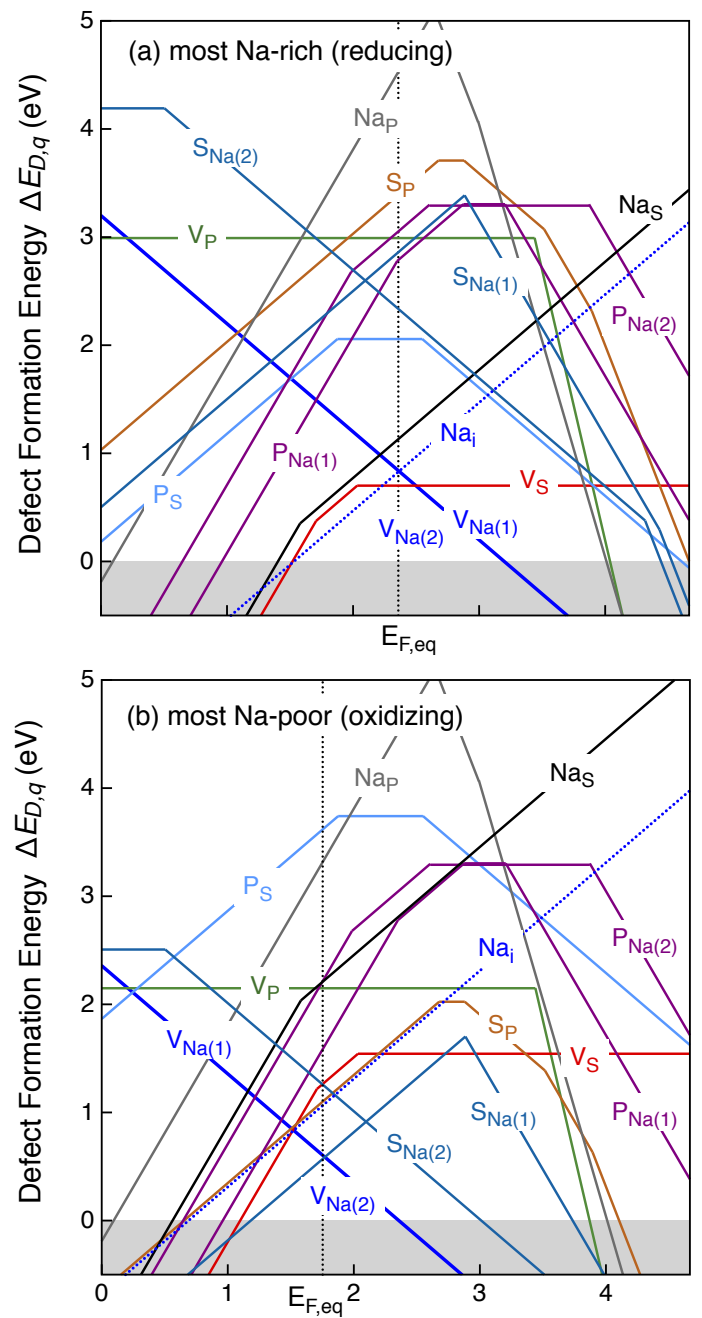

Fermi energy $E_{\mathrm{F}}(\mathrm{eV})$

Figure S2: Formation energies of native point defects $\left(\Delta E_{D, q}\right)$ in tetragonal $\mathrm{Na}_{3} \mathrm{PS}_{4}$ as functions of Fermi energy $\left(E_{\mathrm{F}}\right)$ at elemental chemical potentials corresponding to the (a) most Na-rich, and (b) most Na-poor conditions within the phase stability region (see Table S3). $E_{\mathrm{F}}$ is referenced to the valence band maximum. The upper limit of $E_{\mathrm{F}}$ shown is the conduction band minimum such that $E_{\mathrm{F}}$ values range from 0 to the band gap. Multiple lines of the same color represent the same defect type at different Wyckoff sites. The equilibrium Fermi energy $\left(E_{\mathrm{F}, \text { eq }}\right)$, marked by the vertical line, is calculated at $800 \mathrm{~K}$. 


\section{S3 Phase Stability in Chemical Potential Space}

Tables S1, S2 and S3 report the range of elemental chemical potentials $\left(\Delta \mu_{\mathrm{i}}\right)$ set by specific phase equilibria existing with the solid electrolytes investigated. Some of these equlibria are also shown in the phase diagrams in Figures S3, S4, and Figure 2 in the main text.

Table S1: Vertices of the phase stability region of $\mathrm{Li}_{6} \mathrm{PS}_{5} \mathrm{Cl}$ in the Li-P-S-Cl chemical potential space. Variations of elemental chemical potential $\Delta \mu_{\mathrm{i}}$ are reported in $\mathrm{eV}$.

\begin{tabular}{|c|c|c|c|c|c|}
\hline Vertex & $\Delta \mu_{\mathbf{L i}}$ & $\Delta \mu_{\mathbf{P}}$ & $\Delta \mu_{\mathbf{S}}$ & $\Delta \mu_{\mathbf{C l}}$ & $\begin{array}{c}\text { Phases in equilibrium } \\
\text { with } \mathrm{Li}_{6} \mathrm{PS}_{5} \mathrm{Cl}\end{array}$ \\
\hline V1 & -2.109 & -0.607 & -0.573 & -2.131 & $\mathrm{Li}_{2} \mathrm{PS}_{3}, \mathrm{Li}_{2} \mathrm{~S}, \mathrm{LiCl}$ \\
\hline $\mathrm{V} 2$ & -2.129 & -0.657 & -0.543 & -2.111 & $\mathrm{Li}_{2} \mathrm{PS}_{3}, \mathrm{Li}_{3} \mathrm{PS}_{4}, \mathrm{LiCl}$ \\
\hline $\mathrm{V} 3$ & -2.119 & -0.647 & -0.553 & -2.131 & $\mathrm{Li}_{2} \mathrm{PS}_{3}, \mathrm{Li}_{2} \mathrm{~S}, \mathrm{Li}_{3} \mathrm{PS}_{4}$ \\
\hline $\mathrm{V} 4$ & -2.396 & -2.039 & 0.000 & -1.845 & $\mathrm{~S}, \mathrm{Li}_{2} \mathrm{~S}, \mathrm{LiCl}$ \\
\hline V5 & -2.400 & -2.015 & 0.000 & -1.840 & $\mathrm{~S}, \mathrm{Li}_{3} \mathrm{PS}_{4}, \mathrm{LiCl}$ \\
\hline V6 & -2.396 & -2.029 & 0.000 & -1.854 & $\mathrm{~S}, \mathrm{Li}_{2} \mathrm{~S}, \mathrm{Li}_{3} \mathrm{PS}_{4}$ \\
\hline
\end{tabular}

Table S2: Vertices of the phase stability region of $\mathrm{Li}_{6} \mathrm{PS}_{5} \mathrm{I}$ in the Li-P-S-I chemical potential space. Variations of elemental chemical potentials $\Delta \mu_{\mathrm{i}}$ are reported in $\mathrm{eV}$.

\begin{tabular}{lccccc}
\hline Vertex & $\boldsymbol{\Delta} \mu_{\mathbf{L i}}$ & $\boldsymbol{\Delta} \mu_{\mathbf{P}}$ & $\boldsymbol{\Delta} \mu_{\mathbf{S}}$ & $\boldsymbol{\Delta} \mu_{\mathbf{I}}$ & $\begin{array}{c}\text { Phases in equilibrium } \\
\text { with } \mathbf{L i} 6 \mathbf{P S}\end{array}$ \\
\hline V1 & -2.121 & -0.649 & -0.551 & -0.871 & $\mathrm{Li}_{2} \mathrm{PS}_{3}, \mathrm{Li}_{3} \mathrm{PS}_{4}, \mathrm{LiI}$ \\
V2 & -2.119 & -0.647 & -0.553 & -0.874 & $\mathrm{Li}_{2} \mathrm{PS}_{3}, \mathrm{Li}_{2} \mathrm{~S}, \mathrm{Li}_{3} \mathrm{PS}_{4}$ \\
V3 & -2.118 & -0.641 & -0.556 & -0.874 & $\mathrm{Li}_{2} \mathrm{PS}_{3}, \mathrm{Li} 2 \mathrm{~S}, \mathrm{LiI}$ \\
V4 & -2.395 & -2.031 & 0.000 & -0.596 & $\mathrm{~S}, \mathrm{Li}_{2} \mathrm{~S}, \mathrm{LiI}$ \\
V5 & -2.396 & -2.029 & 0.000 & -0.598 & $\mathrm{~S}, \mathrm{Li}_{2} \mathrm{~S}, \mathrm{Li}_{3} \mathrm{PS}_{4}$ \\
\hline
\end{tabular}

The elemental chemical potentials corresponding to the most Li-rich (reducing) conditions correspond to the vertices V1 and V3 in Tables S1 and S2, respectively. The most Li-poor (oxidizing) conditions are identified by vertices V5 and V6 in Tables S1 and S2, respectively. 
Table S3: Vertices of the phase stability region of $\mathrm{Na}_{3} \mathrm{PS}_{4}$ in the Na-P-S chemical potential space. Variations of elemental chemical potentials $\Delta \mu_{\mathrm{i}}$ are reported in $\mathrm{eV}$.

\begin{tabular}{lcccc}
\hline Vertex & $\boldsymbol{\Delta} \mu_{\mathrm{Na}}$ & $\boldsymbol{\Delta} \mu_{\mathbf{P}}$ & $\boldsymbol{\Delta} \mu_{\mathbf{S}}$ & $\begin{array}{c}\text { Phases in equilibrium } \\
\text { with } \mathrm{Na}_{3} \mathbf{P S}_{4}\end{array}$ \\
\hline V1 & -2.250 & -1.708 & 0.000 & $\mathrm{~S}_{2} \mathrm{Na}_{2} \mathrm{~S}_{5}$ \\
V2 & -2.311 & -1.526 & 0.000 & $\mathrm{~S}, \mathrm{Na}_{2} \mathrm{PS}_{3}$ \\
V3 & -1.799 & -2.238 & -0.206 & ${\mathrm{NaS}, \mathrm{NaS}_{2}}$ \\
V4 & -1.469 & -0.684 & -0.842 & $\mathrm{Na}_{2} \mathrm{~S}_{2} \mathrm{Na}_{2} \mathrm{PS}_{3}$ \\
V5 & -1.775 & -2.214 & -0.230 & $\mathrm{Na}_{2} \mathrm{~S}, \mathrm{NaS}$ \\
V6 & -2.053 & -1.984 & -0.079 & $\mathrm{Na}_{2} \mathrm{~S}_{5}, \mathrm{NaS}_{2}$ \\
\hline
\end{tabular}




\section{S4 Phase Equilibria of $\mathrm{Li}_{6} \mathrm{PS}_{5} \mathrm{Cl}$ and $\mathrm{Na}_{3} \mathrm{PS}_{4}$}

Figure S3 shows projections of the computed quaternary Li-S-P-Cl phase diagrams at specific chemical and electrochemical conditions. Figure S3(a) corresponds to the projection at the most Li-rich condition under which $\mathrm{Li}_{6} \mathrm{PS}_{5} \mathrm{Cl}$ is thermodynamically stable. This also corresponds to thermodynamic stability of $\mathrm{Li}_{6} \mathrm{PS}_{5} \mathrm{Cl}$ in a highly reducing environment set by $\mathrm{Li}_{2}$, which in turn is in equilibrium with Li-metal. Figure $\mathrm{S} 3(\mathrm{~b})$ corresponds to the projection at the most Li-poor condition under which $\mathrm{Li}_{6} \mathrm{PS}_{5} \mathrm{Cl}$ is thermodynamically stable. This also corresponds to thermodynamic stability of $\mathrm{Li}_{6} \mathrm{PS}_{5} \mathrm{Cl}$ in an oxidizing environment.
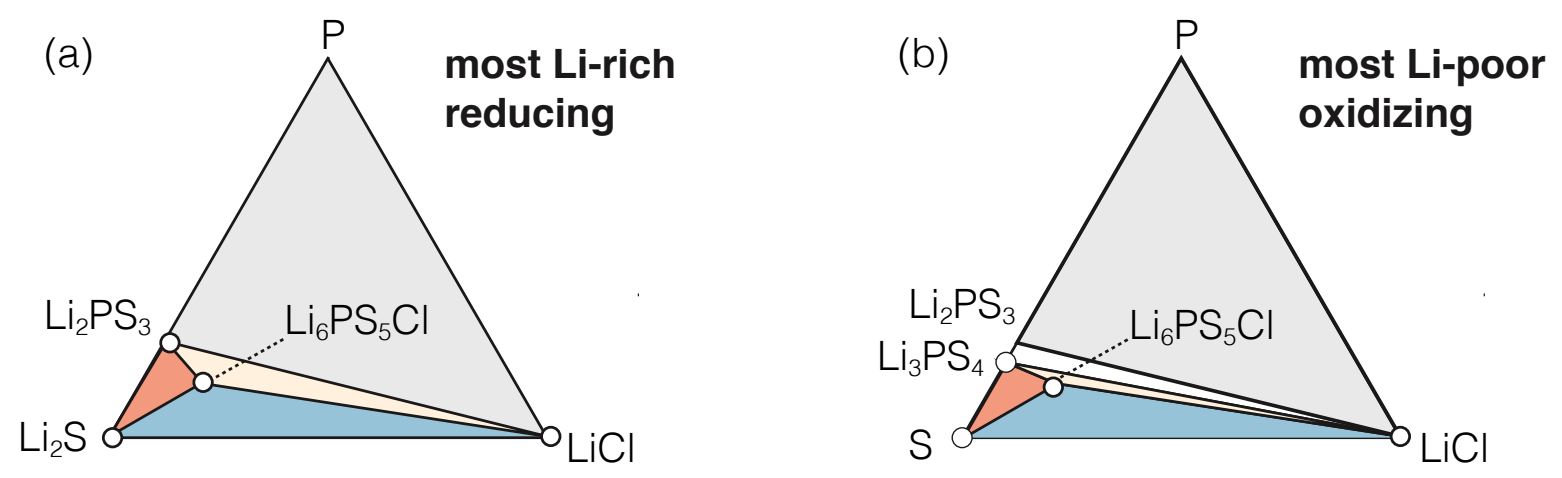

Figure S3: Projected phase diagrams of Li-P-S-Cl at specific Li chemical potentials: (a) Li-rich or reducing conditions, and (b) Li-poor or oxidizing conditions. Equilibrium tie lines connecting $\mathrm{Li}_{6} \mathrm{PS}_{5} \mathrm{Cl}$ to the neighboring competing phases are shown with solid black lines.

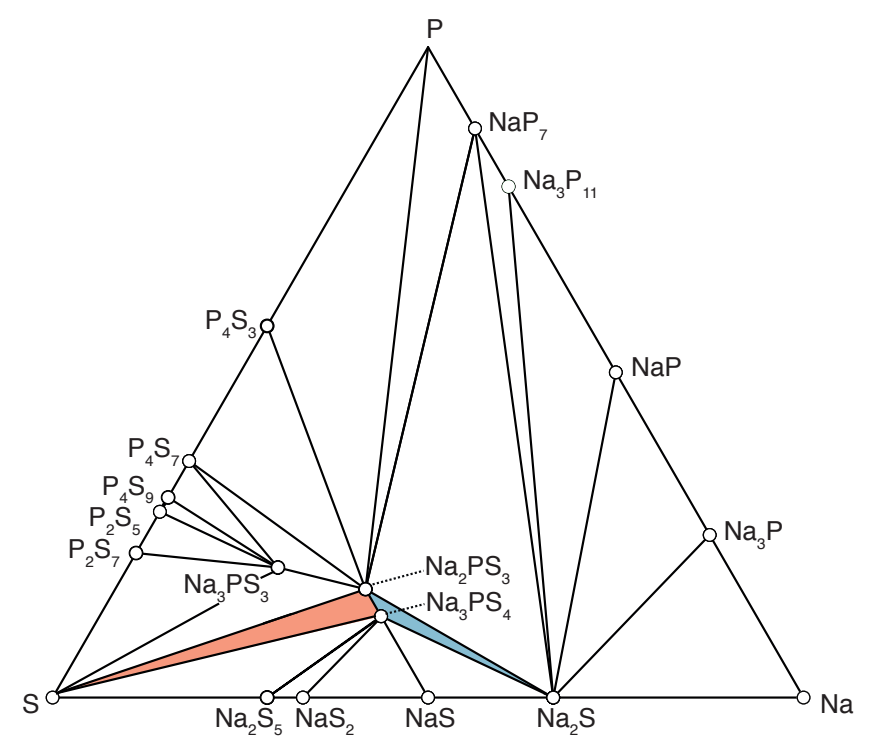

Figure S4: Phase diagram in the ternary Na-P-S chemical space. The three-phase equilibrium regions are highlighted in orange and blue. 


\section{S5 Ensemble Statistics for Modeling Disorder in Argyrodites}

Li argyrodites $\mathrm{Li}_{6} \mathrm{PS}_{5} X\left(X=\mathrm{Cl}\right.$, and I) display structural disorder, ${ }^{1}$ including partial site occupancy. An ensemble statistical approach was employed to model the site disorder in $\operatorname{Li}_{6} \mathrm{PS}_{5} X{ }^{2}$ A disordered macrostate can be expressed as a thermodynamic average of structurally ordered microstates. ${ }^{3,4}$ Symmetrically distinct orderings in $\mathrm{Li}_{6} \mathrm{PS}_{5} X$ were identified using enumlib by Hart and Forcade. ${ }^{5}$ We identified 831 and 1847 unique orderings in the unit cells of $\mathrm{Li}_{6} \mathrm{PS}_{5} \mathrm{Cl}$ and $\mathrm{Li}_{6} \mathrm{PS}_{5} \mathrm{I}$, respectively.

The thermodynamic contribution of each microstate to the disordered structure is proportional to:

$$
g(E) e^{-\frac{\left(E-E_{\min }\right)}{k_{B} T}}
$$

where $E$ is the total energy of a microstate, $g(E)$ is its multiplicity (degenearacy), and $k_{B}$ is the Boltzmann constant. The multiplicity of each state is decided on the basis of equality of DFT total energies. The thermodynamic contribution of each microstate can be expressed as the probability:

$$
\frac{1}{Z} g(E) e^{-\frac{\left(E-E_{\min }\right)}{k_{B} T}}
$$

with $Z$ the partition function. Figures S5 and S6 show the computed probability distribution of the 831 and 1847 structures of $\mathrm{Li}_{6} \mathrm{PS}_{5} \mathrm{I}$ and $\mathrm{Li}_{6} \mathrm{PS}_{5} \mathrm{Cl}$, respectively, as a function of energy per atom. An appropriate condition for selecting representative structures is to choose those with high ensemble probabilities.

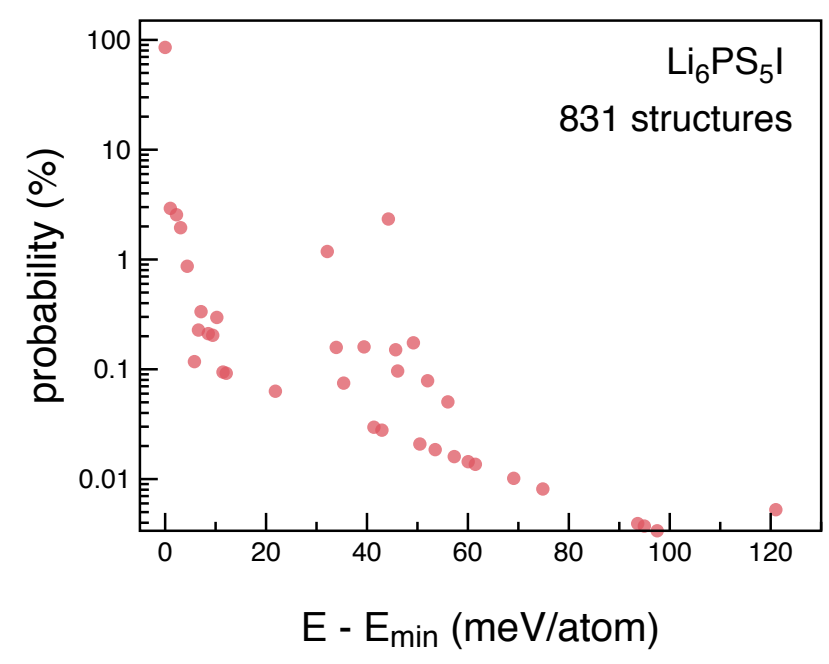

Figure S5: Probability distribution of the 831 relaxed structures of $\mathrm{Li}_{6} \mathrm{PS}_{5} \mathrm{I}$ calculated using ensemble statistics. The relaxed structure with the highest probability $(\sim 85 \%)$ is chosen as a representative structure for disordered $\mathrm{Li}_{6} \mathrm{PS}_{5} \mathrm{I}$. 


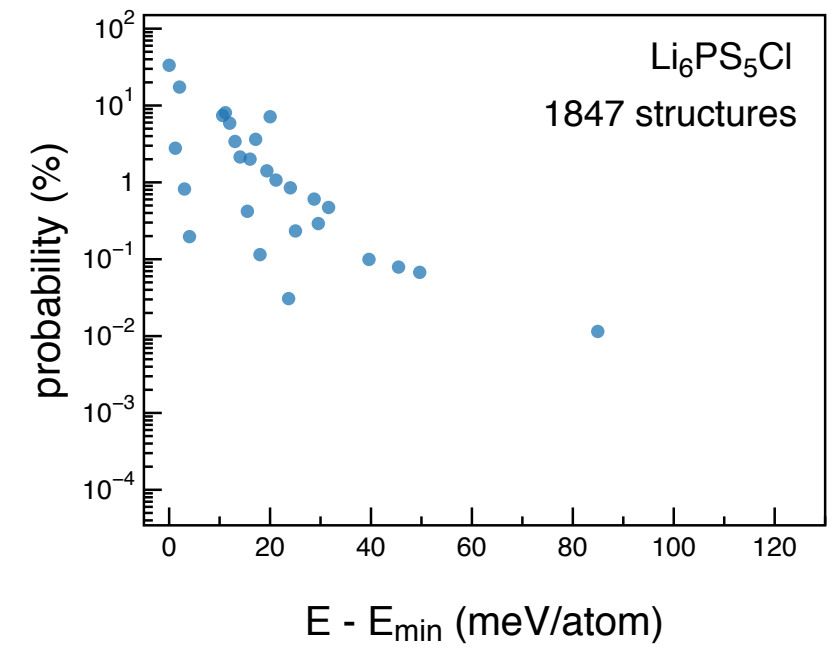

Figure S6: Probability distribution of the 1847 relaxed structures of $\mathrm{Li}_{6} \mathrm{PS}_{5} \mathrm{Cl}$ calculated using ensemble statistics. The relaxed structure with the highest probability $(\sim 33 \%)$ is chosen as a representative structure for disordered $\mathrm{Li}_{6} \mathrm{PS}_{5} \mathrm{Cl}$.

We chose the most probable structures marked in Figures S5 and S6 to perform the defects calculations. 


\section{S6 Crystal Structure of Tetragonal $\mathrm{Na}_{3} \mathrm{PS}_{4}$}

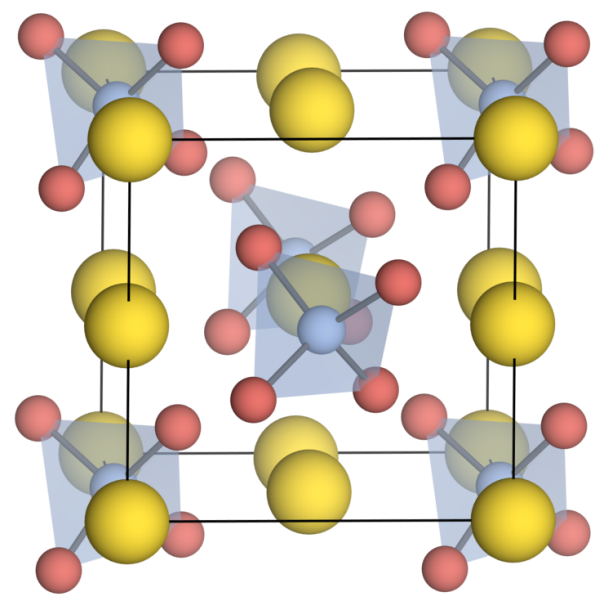

Figure S7: Crystal structure of tetragonal $\mathrm{Na}_{3} \mathrm{PS}_{4}$. 


\section{S7 Band-gap Measurements of the Tetragonal and Cubic $\mathrm{Na}_{3} \mathrm{PS}_{4}$ Phases}

Figure S8 and S9 show the reflectance spectra collected for the high-temperature (HT) tetragonal-Na3 $\mathrm{PS}_{4}$ and the tetragonal-Na $\mathrm{PS}_{4}\left(P \overline{4} 2_{1} c\right)$ obtained from the annealed ball-milled-cubic- $\mathrm{Na}_{3} \mathrm{PS}_{4}$ phase, respectively. Figure S10 shows the reflectance spectra of the ball-milled cubic-Na $\mathrm{PS}_{4}(I \overline{4} 3 m)$.

(a)

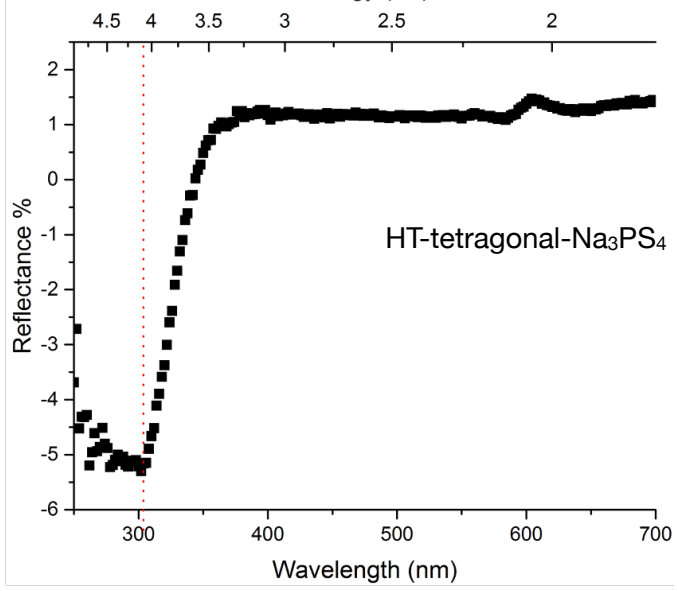

(b)

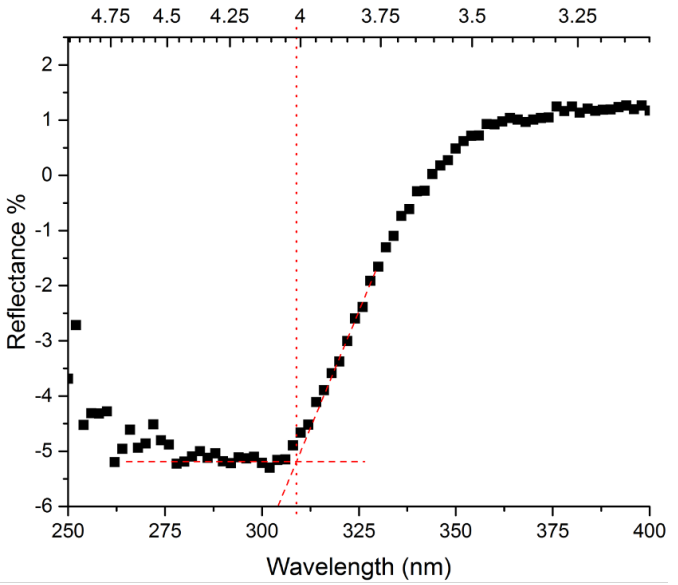

Figure S8: Panel (a) reflectance spectra of the high-temperature (HT) tetragonal $\mathrm{Na}_{3} \mathrm{PS}_{4}\left(P \overline{4} 2_{1} c\right)$. Panel (b) zoom in of the reflectance spectra near the adsorbtion edge, i.e. wavelength range $250-400 \mathrm{~nm}$.
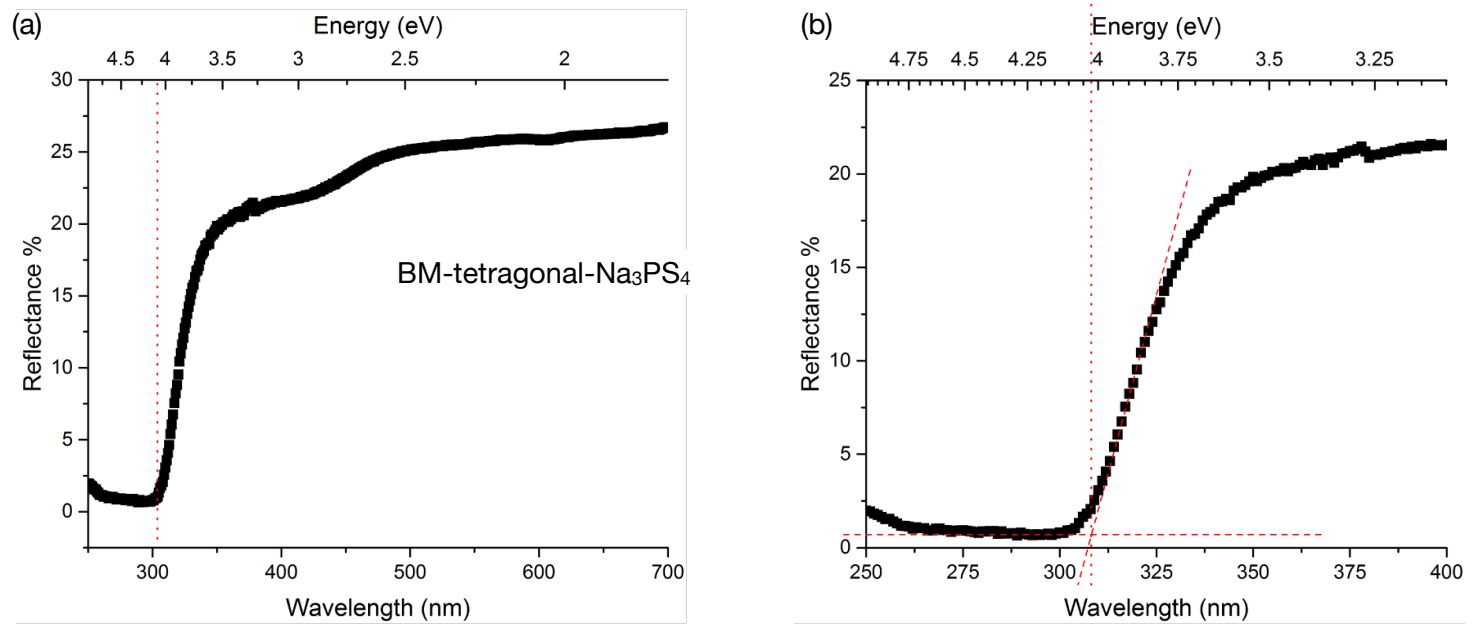

Figure S9: Panel (a) reflectance spectra of the ball-milled (BM) tetragonal- $\mathrm{Na}_{3} \mathrm{PS}_{4}$ obtained after annealing the ball-milled cubic-Na $\mathrm{PS}_{4}$ polymorph. Panel (b) zoom in of the reflectance spectra near the adsorbtion edge, i.e. wavelength range $250-400 \mathrm{~nm}$. 

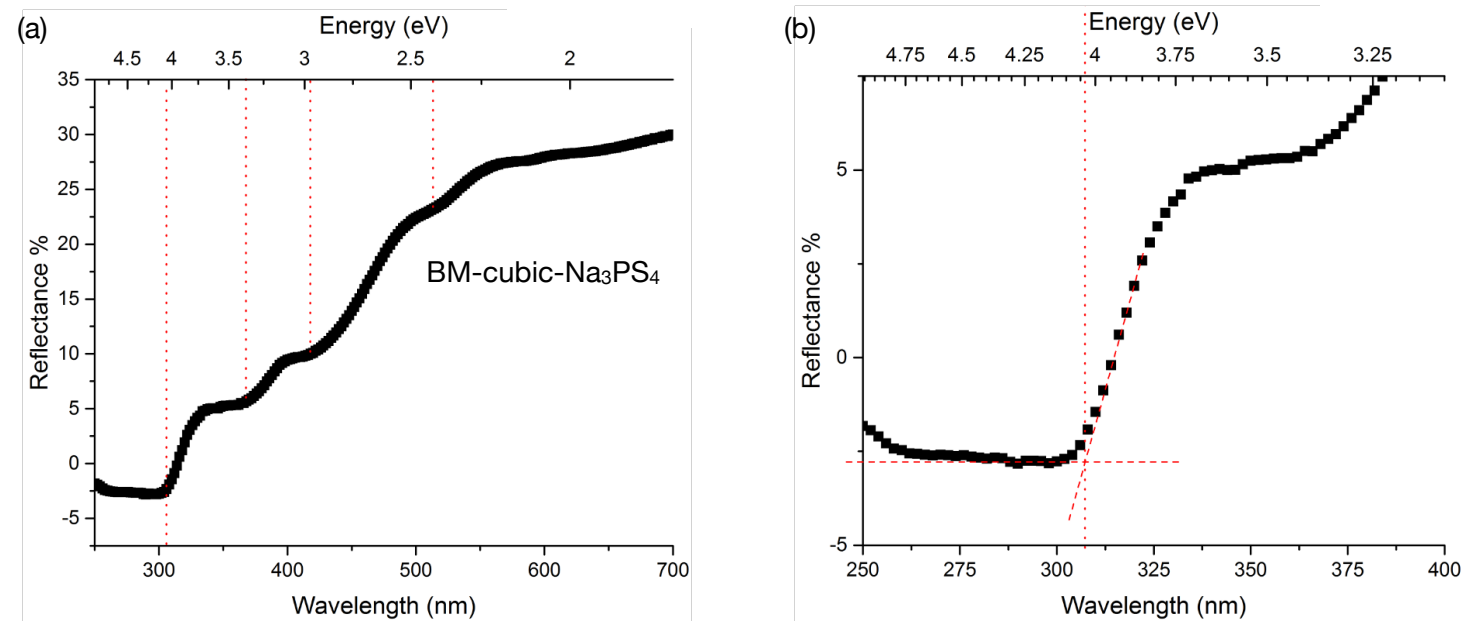

Figure S10: Panel (a) reflectance spectra of the ball-milled (BM) cubic-Na $\mathrm{PS}_{4}(I \overline{4} 3 m)$. Panel (b) zoom in of the reflectance spectra near the adsorbtion edge, i.e. wavelength range $250-400 \mathrm{~nm}$.

Figures S11 shows the Tauc plot of HT tetragonal- $\mathrm{Na}_{3} \mathrm{PS}_{4}$ phase and the band-gap determination. The functional form of the so-called Tauc plot and y-axis of Figures S11 and S13 is defined in Eq. S3.

$$
\sqrt{\mathcal{F}(R) h \nu}=A\left(h \nu-E_{g}\right)
$$

where $h$ is the Plank constant, $\nu$ is the frequency of vibration, $\mathcal{F}(R)$ is the adsrobtion coefficient, $E_{g}$ the band gap and $A$ a proportion constant.
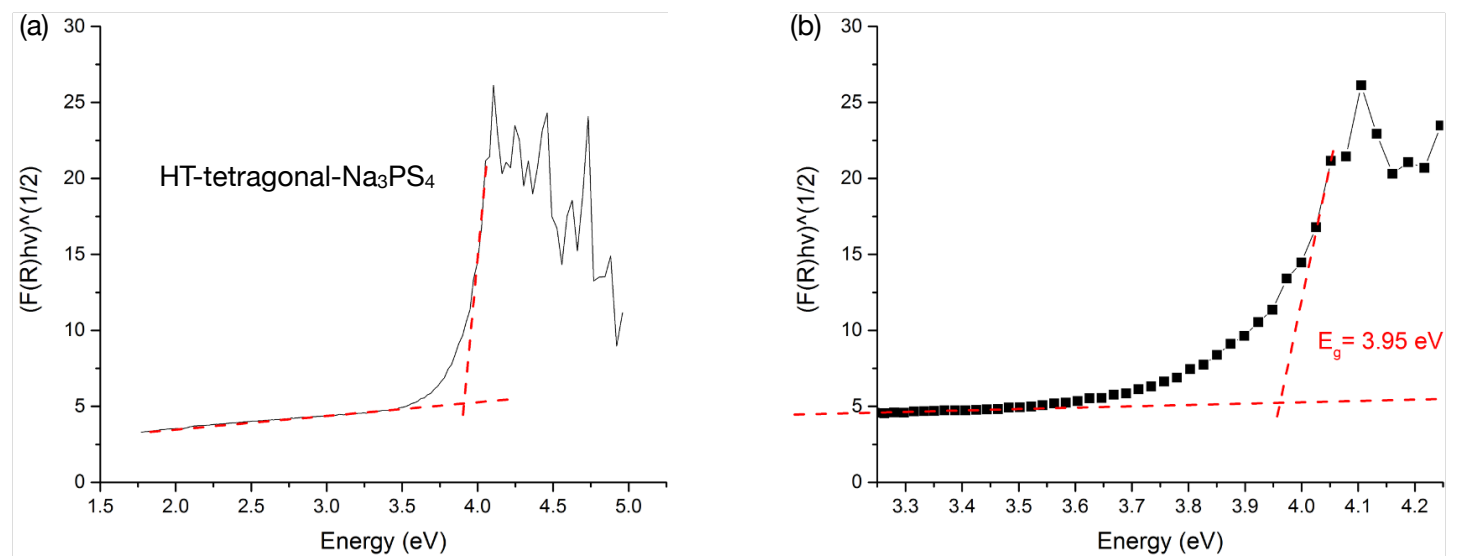

Figure S11: Tauc plot of the HT tetragonal- $\mathrm{Na}_{3} \mathrm{PS}_{4}$ phase. Panel (b) zoom in of the Tauc plot near the adsrobtion edge, i.e. wavelength range $3.25-4.25 \mathrm{eV}$. 
(a)

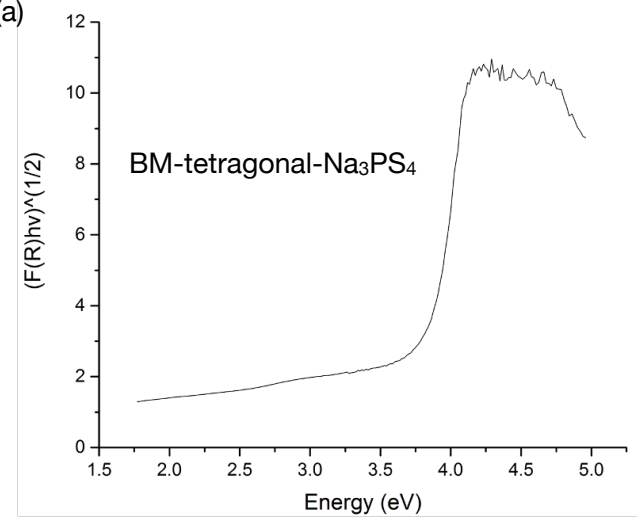

(b)

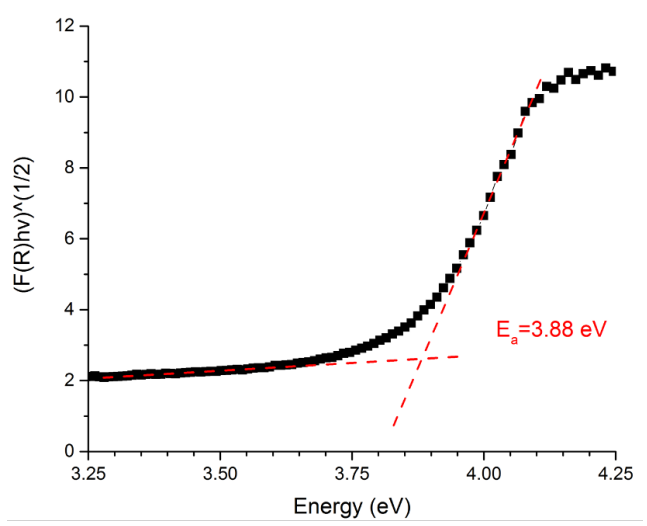

Figure S12: Tauc plot of the ball-milled (BM) tetragonal-Na $\mathrm{PS}_{4}$ obtained after annealing the ball-milled cubic- $\mathrm{Na}_{3} \mathrm{PS}_{4}$ phase. Panel (b) zoom in of the Tauc plot near the adsrobtion edge, i.e. wavelength range 3.25-4.25 eV.
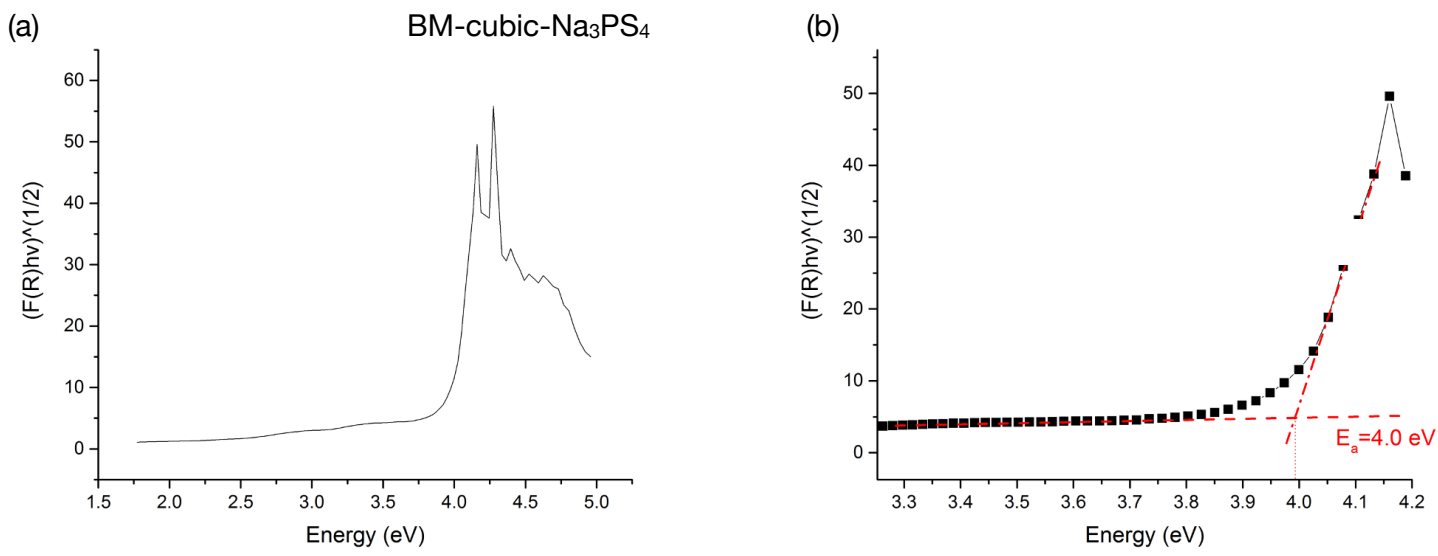

Figure S13: Tauc plot of the ball-milled cubic-Na $\mathrm{PS}_{4}$. Panel (b) zoom in of the Tauc plot near the adsrobtion edge, i.e. wavelength range $3.25-4.20 \mathrm{eV}$.

The determination of the band gap remains entirely dependent on the fitting method used. Table S4 reports band gaps of the $\mathrm{HT}$ and $\mathrm{BM}$ tetragonal- $\mathrm{Na}_{3} \mathrm{PS}_{4}$ assigned with the different methodologies. 
Table S4: Band gap values (in eV) for HT tetragonal- $\mathrm{Na}_{3} \mathrm{PS}_{4}, \mathrm{BM}$ tetragonal- $\mathrm{Na}_{3} \mathrm{PS}_{4}$ and $\mathrm{BM}_{\mathrm{Cubic}-\mathrm{Na}} \mathrm{PS}_{4}$ as determined directly (Direct) from the adsrobtion edge and via the Tauc plot with Kubelka-Munk function. The experimentally determined space groups are also reported.

\begin{tabular}{lccc}
\hline Solid Electrolyte & Space Group & Direct & Tauc \\
\hline HT tetragonal- $\mathrm{Na}_{3} \mathrm{PS}_{4}$ & $P \overline{4} 2_{1} c$ & 4.05 & 3.95 \\
$\mathrm{BM}$ tetragonal-Na $\mathrm{PS}_{4}$ & $P \overline{4} 2_{1} c$ & 4.10 & 3.88 \\
$\mathrm{BM}$ cubic-Na $\mathrm{PS}_{4}$ & $I \overline{4} 3 m$ & 4.10 & 4.00 \\
\hline
\end{tabular}

\section{References}

(1) Kraft, M. A.; Culver, S. P.; Calderon, M.; Böcher, F.; Krauskopf, T.; Senyshyn, A.; Dietrich, C.; Zevalkink, A.; Janek, J.; Zeier, W. G. Influence of Lattice Polarizability on the Ionic Conductivity in the Lithium Superionic Argyrodites $\mathrm{Li}_{6} \mathrm{PS}_{5} \mathrm{X}(\mathrm{X}=\mathrm{Cl}, \mathrm{Br}, \mathrm{I})$. J. Am. Chem. Soc. 2017, 139, 10909-10918.

(2) Gorai, P.; Long, H.; Jones, E.; Santhanagopalan, S.; Stevanović, V. Defect chemistry of disordered solid-state electrolyte $\mathrm{Li}_{10} \mathrm{GeP}_{2} \mathrm{~S}_{12}$. J. Mater. Chem. A 2020, 8, 3851-3858.

(3) Jones, E.; Stevanovic, V. The Glassy Solid as a Statistical Ensemble of Crystalline Microstates. npJ Computational Materials 2020, 6, 56.

(4) Moran, R. F.; McKay, D.; Tornstrom, P. C.; Aziz, A.; Fernandes, A.; Grau-Crespo, R.; Ashbrook, S. E. Ensemble-Based Modeling of the NMR Spectra of Solid Solutions: Cation Disorder in $\mathrm{Y}_{2}(\mathrm{Sn}, \mathrm{Ti})_{2} \mathrm{O}_{7} . J$. Amer. Chem. Soc. 2019, 141, 17838.

(5) Hart, G. L. W.; Forcade, R. W. Algorithm for generating derivative structures. Phys. Rev. B 2008, 77, 224115. 